\title{
UNA METODOLOGIA BASADA EN COPULASY VALORES EXTREMOS PARA ESTIMAR EL CAPITAL ECONOMICO REQUERIDO DE UN PORTAFOLIO DE CREDITOS AL MENUDEO
}

\author{
A COPULA AND EXTREME-VALUE BASED METHODOLOGY \\ FOR ESTIMATING THE REQUIRED ECONOMIC CAPITAL IN A \\ RETAIL-CREDIT PORTFOLIO
}

\section{ADAN DIAZ HERNANDEZ* \\ University of Essex, UK}

\section{JOSE CARLOS RAMIREZ SANCHEZ**}

Universidad Anáhuac y CIDE, México

\begin{abstract}
This paper poses a new methodology to estimate the required economic capital for a retail-credit portfolio. The methodology is based on both the general copula concepts and some core results from the extreme value theory (EVT). The main results support the fact that the proposed methodology is more flexible than other traditional techniques, in particular when it makes use of elliptical generalized or grouped t Student copulas to model the dependence structure of risk-parameters or when it includes elements of the EVT to analyze the extreme losses behavior of a retail-credit portfolio. When applying algorithms, the paper includes data from a Mexican bank.
\end{abstract}

Keywords: Economic capital, credit risk, copulas, extreme value theory.

JEL Classification: $C 14, C 15, C 16, G 32$.

\section{Resumen}

El documento propone una metodología para estimar el capital económico requerido de un portafolio de créditos al menudeo basada en los concep-

\footnotetext{
* University of Essex, UK, y consultor en Administración de Riesgos de varias instituciones financieras de México. E-mail: adiazh@essex.ac.uk

** Profesor-Investigador del CADEN-Universidad Anáhuac México-Norte y profesor afiliado del Centro de Investigación y Docencia Económicas (CIDE). E-mail: jose.ramirez@anahuac.mx
} 
tos generales de cópulas y de la teoría de valores extremos (TVE). Los resultados avalan la mayor flexibilidad de la metodología propuesta sobre algunas técnicas tradicionales, en particular cuando ésta incorpora cópulas elípticas generalizadas y/o agrupadas del tipo t de Student para modelar la estructura de dependencia de los parámetros de riesgo, o cuando hace uso de la TVE para analizar el comportamiento de las pérdidas extremas del portafolio. En la aplicación de los algoritmos se utilizan datos de un banco mexicano.

Palabras Clave: Capital económico requerido, riesgo de crédito, cópulas, valores extremos.

Clasificación JEL: C14, C15, C16, G32.

\section{INTRODUCCION}

No hay duda que una de las tareas más importantes en la administración de las instituciones financieras es la determinación conjunta de las cantidades óptimas de riesgo y capital. La razón es que el uso eficiente del capital involucrado en la operación de cualquier negocio financiero depende, crucialmente, de un sistema adecuado de valuación de riesgos. Sin el cálculo correcto del monto de capital en riesgo, o capital susceptible de ser perdido en un escenario desfavorable, no es posible estimar realistamente el costo alternativo de la inversión de los accionistas.

El problema es que mientras las mediciones de riesgo son puntuales y objetivas, las funciones de administración y distribución del capital son una mezcla de ciencia, experiencia y arte (Saita, 2007). En la práctica no hay algoritmos mágicos ni recetas universales que permitan conocer, de manera infalible, los montos óptimos del capital ajustado por riesgo. Hay, a lo sumo, lineamientos generales, derivados de controles internos o disposiciones regulatorias que buscan darles a los administradores una idea del balance que existe entre sus estimaciones internas de capital y sus necesidades de protección contra riesgos por pérdidas potenciales.

Los lineamientos regularmente usados para estimar el capital en riesgo buscan definir, primero, el concepto de capital que está bajo consideración y, luego, las medidas de riesgo asociadas a las operaciones cubiertas por ese capital (Matten, 2000). En el primer caso, la definición requiere de distinguir entre capital regulatorio $(\mathrm{CR})$ y capital económico (CE), ya que si bien ambos guardan una estrecha correspondencia entre sí no son conceptos intercambiables. Mientras que el CE es el capital en riesgo estimado por la institución para asegurar la viabilidad de sus líneas de negocios, el CR es calculado con base en las disposiciones y metodologías de las autoridades reguladoras. Debido a su distinto origen y, en particular, al hecho de que las instituciones financieras incluyen a menudo clases de riesgos que no necesariamente están contempladas en el CR, ambos montos suelen diferir. Por esa razón se recomienda utilizar el concepto de capital económico requerido $(C E R)$, o capital en riesgo estimado con independencia de las restricciones regulatorias, ya que a la vez que ofrece una 
idea más comprensiva y realista de las necesidades diarias de las instituciones para cubrirse de los riesgos, contempla los requerimientos mínimos del capital regulatorio (Saita, 2007). Como es de esperarse, el CER no necesariamente coincide con el capital disponible de la institución.

La estimación del CER varía de acuerdo con cada una de las siguientes nociones de capital adoptadas como base, esto es: como valor en libros, valor de mercado o capitalización de mercado. La variabilidad en cada caso puede ser muy pronunciada, por lo que la elección adecuada de tal o cual noción depende críticamente de la naturaleza de las posiciones y del tipo de riesgo bajo consideración.

En el cálculo del CER de un portafolio de crédito, por ejemplo, la cantidad fijada como capital en riesgo está determinada por las pérdidas inesperadas de sus posiciones que, a su vez, constituyen una medida de su valor en riesgo. Pero el monto de las pérdidas es muy distinto si se utiliza un enfoque de valor de mercado (mark-tomarket) que uno basado en el valor en libros (book-value accounting). En el primero, el valor de las posiciones sensibles al riesgo es equivalente al valor presente, a precios de mercado, de sus sumas de efectivo descontadas conforme a una tasa ajustada por riesgo. De esta manera, cualquier disminución en el valor presente del portafolio, por efecto digamos de un aumento en la tasa de descuento, se contabilizaría como pérdidas inesperadas. En el segundo enfoque, en cambio, las pérdidas estarían asociadas únicamente a la baja reconocida por las instituciones en el valor en libros de sus posiciones. Las diferencias entre ambos enfoques se traducirían en diferencias en las causas de las pérdidas, pues mientras que una degradación en las calificaciones del acreedor dejaría inafectado el cálculo del $C E R$ en valor en libros, en el enfoque de valor de mercado ésta conduciría a mayores márgenes de crédito y, en consecuencia, a un menor valor de mercado de las posiciones.

La correcta especificación del CER no significa, empero, el fin del problema de su estimación sino su inicio. Y es que una vez decidida la noción de capital queda todavía pendiente por seleccionar el mejor modelo de estimación del riesgo que nos garantice el cálculo más adecuado del monto del CER; y éste no es un asunto menor. En el ejemplo del riesgo de crédito que mencionamos arriba hay que enfrentar, para empezar, una serie de obstáculos relacionada con la tipificación del portafolio, la estructura de dependencia de los parámetros de riesgo y, finalmente, la confirmación de la superioridad y robustez de las estimaciones.

Como se sabe, en la literatura existe una amplia gama de trabajos que permiten modelar el riesgo de crédito de portafolios de préstamos comerciales -empresas o personas morales- de acuerdo con los lineamientos propuestos por CreditMetrics (CM), KMV PortfolioManager (KMV), CreditRisk+, o CreditPortfolioView (véase Crouhy et al., 2000; y Crosbie y Bohn, 2002). Pero ésta no parece ser la misma situación para los préstamos a personas físicas. La escasez de trabajos en este rubro ha obligado a los practicantes a adaptar los modelos de préstamos comerciales para medir el riesgo de crédito en portafolios de préstamos personales, según sean las necesidades o las disponibilidades de información de las instituciones financieras (De Andrade y Thomas, 2004; y Perli y Nayda, 2004). De hecho, las reglas de riesgo de crédito establecidas por Basilea II en su enfoque más avanzado de calificaciones internas (IRB) tienen 
sus bases sobre ciertos resultados asintóticos para portafolios grandes bajo versiones simplificadas de los modelos del tipo KMV/CM.

Del mismo modo, hay diversas maneras de relacionar los parámetros de riesgo que determinan las pérdidas del portafolio atribuidas al riesgo de crédito -probabilidad de incumplimiento (PD), exposición al incumplimiento (EAD) y pérdida dado el cumplimiento (LGD) - y no todas con los mismos resultados. La adopción de distintas estructuras de dependencia entre los parámetros de riesgo genera, a su vez, distintas distribuciones de pérdidas simuladas y, por ende, diferentes montos de CER.

Por todas estas razones, es claro que el cálculo adecuado del $C E R$ requiere de metodologías que sean conceptual y estadísticamente robustas en cada una de sus etapas. La tarea no es, de ninguna manera, fácil ya que lejos de observar en la práctica una utilización homogénea de conceptos y técnicas en la medición de riesgo de crédito, las instituciones financieras han tendido a ajustar discrecionalmente sus estimaciones a los distintos enfoques estipulados por Basilea II. Como resultado, el cálculo del $C E R$ de las posiciones de crédito se ha convertido más bien en un ejercicio regido por factores idiosincrásicos que en una práctica común controlada por disposiciones generales a la industria. Al menos esa es la experiencia de los bancos mexicanos y norteamericanos (Márquez y López, 2006; y Saita, 2007).

El objetivo principal de este documento es proponer una metodología orientada a facilitar el cómputo del CER de un portafolio de créditos al menudeo -o de personas físicas-en instituciones financieras como las mexicanas. Para tal efecto, la propuesta busca simultáneamente: 1) establecer la relación entre los parámetros de riesgo mediante el uso de cópulas multivariadas y medir su efectividad frente a otras estructuras de dependencia que han recibido mayor atención en la literatura; 2) aplicar algunos resultados de la Teoría de Valores Extremos (TVE), relacionados con el método de Peaks Over Threshold (POT), para estimar, primero, el Valor en Riesgo $\left(V_{a} R_{q}\right)$, el Déficit Esperado $\left(E S_{q}\right)$ y, luego, el $C E R$ del portafolio; 3) comparar la robustez de la metodología con otras consagradas por la práctica mediante pruebas bilineales de bondad de ajuste; y finalmente 4) evaluar el impacto que tienen las reglas de capitalización propuestas en el enfoque más avanzado de $I R B$ sobre los requerimientos de capital del portafolio.

Los resultados del documento son, en cierta manera, novedosos pues no hay antecedentes en la literatura local en los que se muestre conjuntamente las siguientes tres características de la metodología aquí propuesta. ${ }^{1}$ La primera es el uso comparativo de cópulas multivariadas para ajustar el mejor modelo de dependencia no sólo entre los parámetros de riesgo de un grupo particular de créditos sino, también, entre los grupos de créditos y productos constitutivos del portafolio. La segunda es el análisis de las bondades de la diversificación de un portafolio que incluye productos con diferentes índices de dependencia extrema y que no es posible apreciar, por ejemplo, en el

1 El único modelo de riesgo de crédito que puede considerarse, por su naturaleza y orientación, un antecedente de este documento está contenido en el excelente libro de Márquez (2006). Sin embargo, el método de estimación que este autor propone está diseñado para préstamos comerciales y con un nivel de agregación nacional que no guarda correspondencia estrecha con el contemplado por nuestra metodología. 
modelo de un factor del tipo KMV/CM; y, finalmente, la tercera es el procedimiento para constatar que las estimaciones del CER de portafolios de crédito al menudeo son, en general, distintas (y en su mayoría superiores) al requerimiento regulatorio establecido por las reglas de capitalización del enfoque más avanzado de IRB.

El resto del documento está organizado de la siguiente manera. La sección II presenta las características del portafolio, su técnica de segmentación y la estimación de sus parámetros de riesgo. La sección III muestra los resultados obtenidos con el uso de las cópulas gaussiana, $t$ de Student simétrica, $t$ de Student generalizada y $t$ de Student agrupada y sus respectivos análisis de bondad de ajuste. La sección IV incluye pruebas de contraste para comparar la robustez de los resultados de la metodología con algunos modelos alternativos consagrados en la práctica, tales como el modelo de incumplimiento de KMV/CM y el enfoque más avanzado de IRB propuesto por Basilea II. Finalmente, las conclusiones exponen los resultados más relevantes. Los algoritmos y resultados técnicos utilizados en las estimaciones aparecen en el Apéndice, al final del documento.

\section{DESCRIPCION DEL PORTAFOLIO Y ESTIMACION DE LOS PARAMETROS DE RIESGO}

\subsection{Descripción y segmentación del portafolio}

Con el propósito de enfatizar el sentido práctico de la metodología aquí propuesta se considera un portafolio de créditos al menudeo dividido en las tres subclases de activos usualmente identificadas por las instituciones bancarias de México; a saber: las exposiciones aseguradas por la vivienda del acreditado (subportafolio hipotecario), las exposiciones revolventes (subportafolio de tarjetas de crédito) y todas las demás exposiciones (subportafolio de créditos al consumo). La información está referida a las operaciones crediticias en valor en libros registradas por un banco mexicano en el período comprendido entre 2001 y $2006 .^{2}$

Debido a que el número de exposiciones es considerablemente grande y a que, en consecuencia, es casi imposible modelar los incumplimientos por cuenta individual, utilizamos la técnica de segmentación Detección de Interacción Automática Ji-Cuadrada (CHAID) para homogeneizar los productos en grupos de créditos de acuerdo con su perfil de riesgo. La técnica es una de las más socorridas en la literatura no sólo por su economía de procedimiento sino, fundamentalmente, por su notable flexibilidad para identificar divisiones óptimas cuando las variables son continuas, nominales e incluso categóricas (Biggs et al., 1991). En conformidad con CHAID, las trayectorias descritas por las variables independientes a lo largo de los árboles de decisión permiten dividir a la población en grupos significativamente diferentes de acuerdo con alguna variable clave propuesta.

2 Por razones de confidencialidad se ha decidido mantener en secreto el origen de la fuente de información. Esta limitación no afecta, sin embargo, el alcance de los resultados ya que el método propuesto puede replicarse sin ninguna pérdida de generalidad en cualquier institución financiera. 
La Tabla 1 muestra los resultados de la segmentación al utilizar a $P D$ como variable clave y al producto, número de pagos vencidos, tiempo en libros, uso de línea de crédito y morosidad observada como variables independientes. ${ }^{3}$ El portafolio resultante de la segmentación arroja 62 subgrupos (o cubetas) repartidos en 10 productos, cada uno con sus respectivos volúmenes (números de cuentas) y montos de exposición (saldos en pesos). Los productos quedan, a su vez, clasificados según los tipos de créditos de la siguiente forma: en créditos al consumo se encuentran las cuentas personales de nómina (productos A y B), los programas especiales del gobierno y pensiones (producto C), así como los productos relacionados con la adquisición de autos (D), bienes del consumidor a pagos fijos (E) y seguros $(\mathrm{F})$; en créditos revolventes se incluyen a las tarjetas de crédito (producto $\mathrm{G}$ ) y, finalmente, en créditos hipotecarios se comprende a los productos indizados en UDIS y con reestructura $(\mathrm{H})$, sin reestructura $(\mathrm{I})$ y con esquemas de interés social $(\mathbf{J})$.

\section{TABLA 1}

PRODUCTOS Y SUBGRUPOS DEL PORTAFOLIO DE CREDITOS

\begin{tabular}{|c|c|c|c|}
\hline Producto & Subgrupos & Número de cuentas & $\begin{array}{c}\text { Exposición* } \\
\text { (MXN millones) }\end{array}$ \\
\hline A & 4 & 2.077 & $\$ 52$ \\
B & 6 & 455.902 & $\$ 1.914$ \\
C & 9 & 25.465 & $\$ 1.343$ \\
D & 8 & 265.835 & $\$ 16.305$ \\
E & 5 & 90.373 & $\$ 1.015$ \\
F & 7 & 2.206 .829 & 387 \\
G & 12 & 9.451 & $\$ 33.519$ \\
H & 4 & 20.642 & $\$ 5.846$ \\
I & 3 & 7.897 & $\$ 10.976$ \\
J & 4 & 3.176 .832 & 796 \\
\hline Total & 62 &
\end{tabular}

* Los datos del producto G constituyen el límite de la línea de crédito.

3 Las variables seleccionadas son ampliamente utilizadas por los practicantes de la industria (véase RMA, 2003). Cabe aclarar, sin embargo, que el objetivo de la metodología no depende del método de segmentación ni de las variables claves intervinientes: la validez de su aplicabilidad es independiente de las técnicas de segmentación empleadas. 


\subsection{Estimación de los parámetros de riesgo}

Una vez segmentado el portafolio, lo que procede es hacer algunas precisiones sobre la notación y el método de cómputo utilizados en la estimación de los parámetros de riesgo. En concreto, identificaremos a $i=1, \ldots, M$ como los grupos o cubetas en los que se divide el portafolio $(M=62)$; a $t=1, \ldots, n$ como las fechas de observación; a $j=1, \ldots, N_{i t}$ y $e_{i t}^{(j)}$ como los créditos y su exposición respectivamente; a $Y_{i t}^{\left({ }^{(j)}\right.}$ como la indicadora del incumplimiento para cada crédito $j$ dentro de una ventana anual a partir de la fecha de observación y, finalmente, a $e a d_{i t}{ }^{(j)}$ como el saldo de dicho crédito al momento de su incumplimiento. El cómputo de los parámetros por grupos o productos considera, por su parte, las siguientes especificaciones:

* Probabilidad de incumplimiento del grupo $\boldsymbol{i}\left(\boldsymbol{P} \boldsymbol{D}_{i}\right)$. En el cálculo de $P D_{i}$ se utiliza a la tasa de incumplimiento observada $T I_{i t}$ como el estimador correspondiente a la ventana anual $t$ (vista a partir de la fecha de observación $t$ ) en el grupo $i$; es decir:

$$
\hat{T}_{i t}=\frac{n_{i t}}{N_{i t}}
$$

donde $n_{i t}=\sum_{i=1}^{N_{i t}} Y_{i t}^{(j)}$ es el número de incumplimientos en la ventana de observación y $T I_{i 1}, \ldots, T I_{i n}$ las realizaciones de $P D_{i}$ para cada cubeta $i=1, \ldots, M$.

* Exposición al momento del incumplimiento del grupo $\boldsymbol{i}\left(\boldsymbol{E A D _ { i }}\right)$. Por razones de simplicidad se seguirá la práctica extendida de estimar este parámetro con el factor de conversión de crédito $C C F_{i}$, que es definido como la proporción perdida de la exposición inicial de los créditos al momento del incumplimiento. El estimador insesgado de la proxy $C C F_{i}$ para la ventana de observación $t$ está dado por:

$$
\hat{C} \hat{C} F_{i t}=\frac{\sum_{j=1}^{N_{i t}} e a d_{i t}^{(j)} Y_{i t}^{(j)}}{\sum_{j=1}^{N_{i t}} e_{i t}^{(j)} Y_{i t}^{(j)}},
$$

* Pérdida dado el incumplimiento por producto $\left(L G D_{p}\right)$. En el cálculo de este parámetro se incluye para cada producto $p$ el porcentaje que se recupera del saldo $e a d_{p t}$ de todos los créditos incumplidos en $t$ durante los primeros $k$ meses posteriores (ventana de recuperación) pues, de otra manera, no se podría estimar la correspondiente tasa de recuperación $\tau_{p t}$. En caso que se incluyan los $\operatorname{costos}$ fijos $c_{p}$ en el proceso de recuperación de cada producto $p$, el estimador de $\tau_{p t}$ estaría dado por

$$
\hat{\tau}_{p t}=\sum_{\tau=1}^{k} \frac{1-c_{p t}^{(\tau)}}{(1+r)^{\tau}} \frac{R_{p t}^{(\tau)}}{e a d_{p t}},
$$


donde $R_{p t}{ }^{(\tau)}$ es la recuperación del mes $\tau$ correspondiente a los créditos del producto $p=1, \ldots, K$ que incumplieron en $t ; c_{i k}{ }^{(j)}$ es el factor de costos y $r$ es la tasa de interés adecuada para descontar los flujos. Con el cálculo de $\tau_{p t}$ se puede obtener, para cada fecha de observación $t=1, \ldots, n$, el estimador

$$
L \hat{G} D_{p t}=1-\hat{\tau}_{p t}
$$

como una realización de $L G D_{p}$ o de la pérdida dado el incumplimiento para el producto $p$ en una ventana de recuperación de $k$ meses. En el cómputo supondremos que $k=24$ meses para el caso de los subportafolios de consumo y tarjetas de créditos y $k=36$ meses para el de hipotecario. Los tamaños de las ventanas de recuperación son fijados a conveniencia para que las estimaciones $\hat{\tau}_{p t}$ se estabilicen a partir de $k$ o más observaciones.

Los resultados de la consolidación de los parámetros de riesgo para los productos A y B así como las estimaciones históricas de $P D, C C F$ y $L G D_{p}$ (este último en histogramas) son expuestos, a manera de ilustración, en los recuadros de la Figura 1. Ahí queda clara la acentuada diferenciación de los valores de $P D$ por cubeta y, en particular, la marcada heterogeneidad de comportamientos distribucionales entre los histogramas de los dos productos. La variabilidad de sus estimaciones es confirmada por los valores de los coeficientes de variación $(C V)$ que fueron construidos en la Tabla 2, primero, para $P D$ en aquellos subgrupos de crédito cuya tasa de incumplimiento observada resultó menor que uno y, luego, para los parámetros agregados por nivel de producto. De acuerdo con esos valores, la variación de las estimaciones de los parámetros no guarda un patrón homogéneo por subgrupo (cuando se considera $P D$ ) ni una correspondencia clara entre los parámetros de cada producto, en tanto que las estimaciones de $C C F$ presentan una variabilidad menor que $P D$ y $L G D$. Estas diferencias en las variaciones alertan sobre la importancia de justificar adecuadamente la estructura de dependencia entre los parámetros ya que, de lo contrario, no habría manera de garantizar una correcta estimación de las medidas de riesgo del portafolio.

Para comprobar este último punto basta realizar el simple ejercicio de evaluar la calidad de las medidas de riesgo resultantes de agregar linealmente el producto de las estimaciones de $P D, E A D$ y $L G D$ sobre la composición (cubetas y productos) del portafolio a fin de obtener las pérdidas históricas (calculadas como porcentaje de saldo). La importancia de hacer un ejercicio de esta naturaleza, que no es más que una aplicación de las ecuaciones (8) y (9) descritas con detalle más adelante, radica en que los valores de sus medidas de riesgo pueden ser tomados como un benchmark histórico mínimo. La Tabla 3 y la Figura 2 resumen este ejercicio considerando la convención usada a lo largo del documento de resaltar en color gris los cálculos hechos con un nivel de confianza del 99,5\% para, así, destacar las medidas de riesgo de las instituciones financieras que aspiran a obtener la más alta calificación crediticia en el país (calificación AAA). Los datos confirman que, en efecto, una pobre estructura de dependencia entre los parámetros, como la ofrecida por un ajuste lineal, arroja 


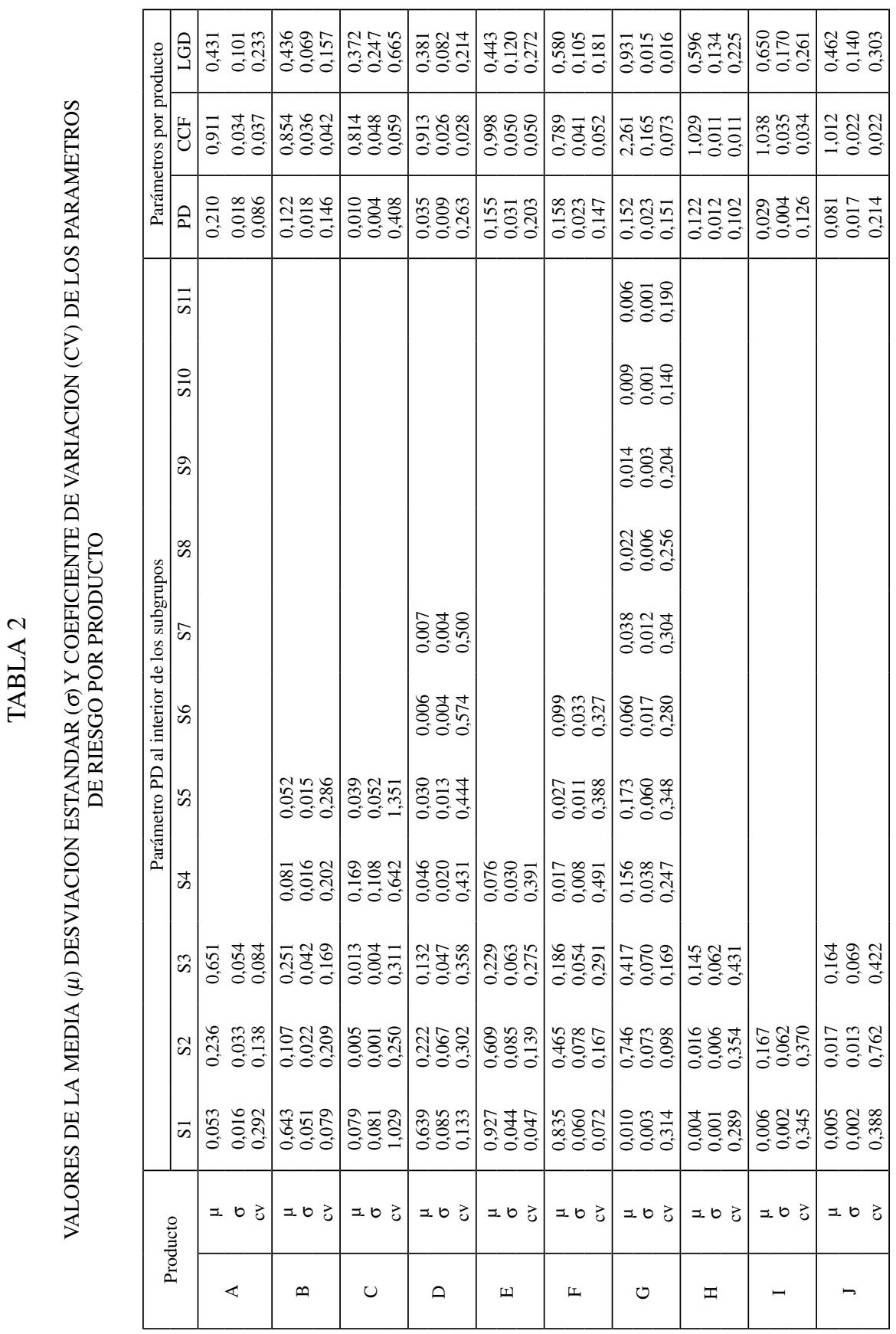




\section{FIGURA 1}

\section{ESTIMACIONES DE PD (IZQUIERDA), CCF (CENTRO) Y LGD (DERECHA)} PARA LOS PRODUCTOS A Y B
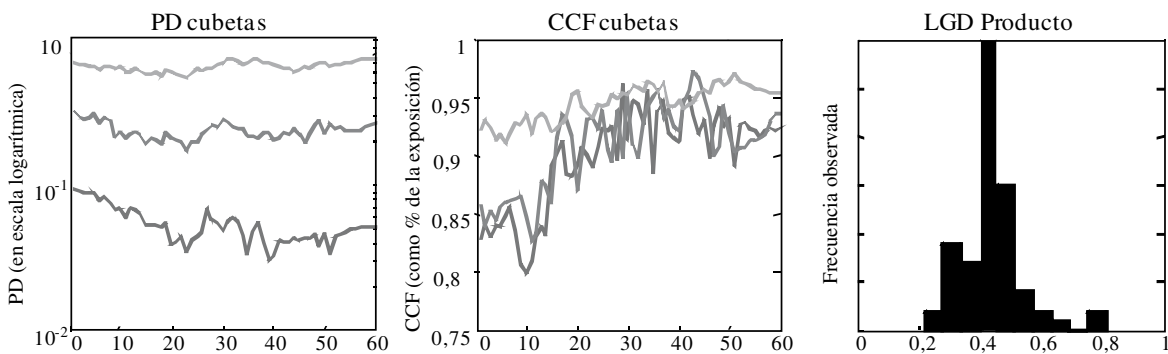

Parámetros de riesgo Producto A
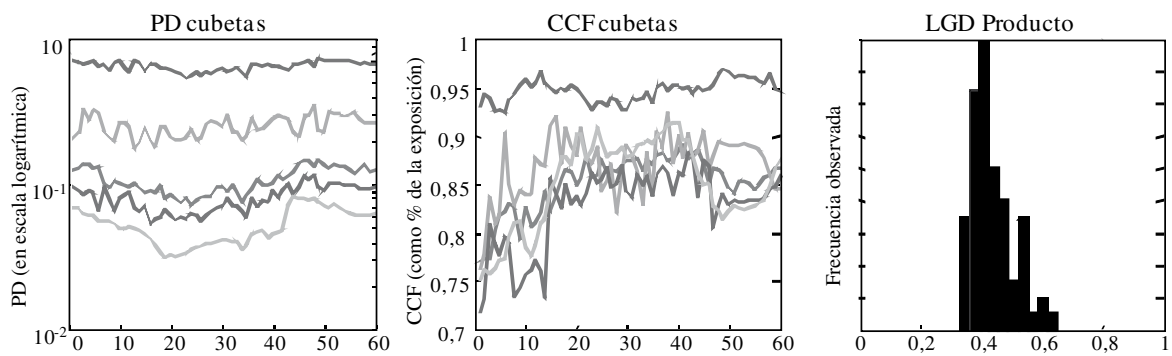

Parámetros de riesgo Producto $\mathrm{B}$

\section{TABLA 3}

ESTIMACIONES EMPIRICAS DE $P E$ Y $V a R q$ POR PRODUCTO Y PORTAFOLIO TOTAL (Benchmark o método histórico)

\begin{tabular}{|c|c|r|r|r|r|}
\hline \multirow{2}{*}{ Producto } & \multirow{2}{*}{ PE } & \multicolumn{4}{|c|}{ VaRqEmp } \\
\cline { 3 - 6 } & & $99 \%$ & $99,5 \%$ & $99,9 \%$ & $99,95 \%$ \\
\hline A & $8,87 \%$ & $10,35 \%$ & $10,37 \%$ & $10,37 \%$ & $10,37 \%$ \\
B & $5,31 \%$ & $6,72 \%$ & $6,73 \%$ & $6,73 \%$ & $6,73 \%$ \\
C & $0,34 \%$ & $0,89 \%$ & $0,90 \%$ & $0,90 \%$ & $0,90 \%$ \\
D & $1,34 \%$ & $2,50 \%$ & $2,51 \%$ & $2,51 \%$ & $2,51 \%$ \\
E & $7,35 \%$ & $11,15 \%$ & $11,15 \%$ & $11,15 \%$ & $11,15 \%$ \\
F & $8,95 \%$ & $12,05 \%$ & $12,10 \%$ & $12,10 \%$ & $12,10 \%$ \\
G & $9,56 \%$ & $12,92 \%$ & $12,93 \%$ & $12,93 \%$ & $12,93 \%$ \\
H & $5,99 \%$ & $7,76 \%$ & $7,76 \%$ & $7,76 \%$ & $7,76 \%$ \\
I & $1,71 \%$ & $2,23 \%$ & $2,23 \%$ & $2,23 \%$ & $2,23 \%$ \\
J & $2,88 \%$ & $4,88 \%$ & $4,88 \%$ & $4,88 \%$ & $4,88 \%$ \\
\hline Total & $3,79 \%$ & $5,21 \%$ & $5,22 \%$ & $5,22 \%$ & $5,22 \%$ \\
\hline
\end{tabular}




\section{FIGURA 2}

HISTOGRAMAS DE LAS PERDIDAS HISTORICAS POR PRODUCTO Y PORTAFOLIO TOTAL

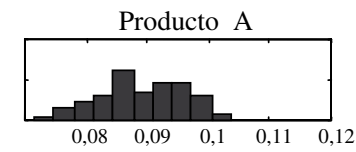

Producto D

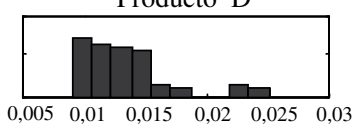

Producto $\mathrm{G}$

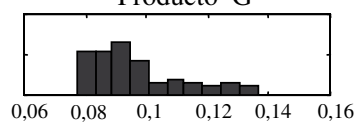

Producto J

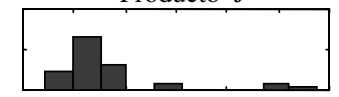

$0,02 \quad 0,0250,03 \quad 0,035 \quad 0,04 \quad 0,0450,05$

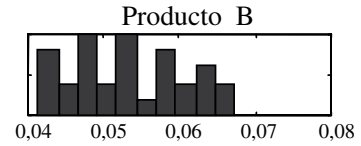

Producto E

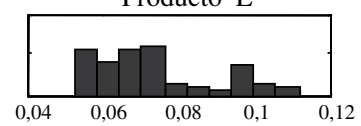

Producto $\mathrm{H}$

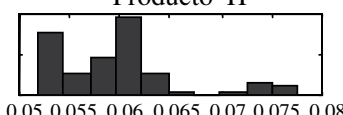

Portafolio Total

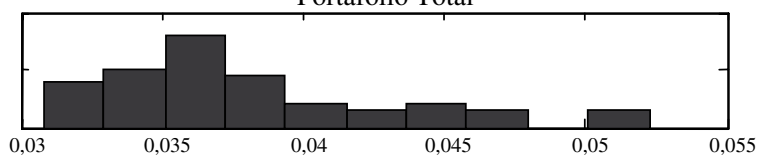

estimaciones empíricas poco robustas sobre el $\operatorname{VaR}_{q}$ y las pérdidas agregadas por producto y portafolio. En concreto, las últimas tres columnas de la Tabla 3 reportan valores de $\operatorname{VaR}_{q}$ que son insensibles a los diferentes niveles de confianza, debido a que son estimadores sesgados de las pérdidas extremas del portafolio que subestiman la cola de la distribución subyacente de pérdidas. La irregularidad en el comportamiento de las pérdidas descrita por los histogramas de la Figura 2 corrobora la pobre calidad de las estimaciones. ${ }^{4}$

En resumen, el ejercicio sugiere que para establecer la correcta estructura de dependencia entre los parámetros es importante hacer, previamente, tratamientos correctivos en las distribuciones de pérdidas históricas para, luego, aplicar métodos estadísticos sobre los extremos de las colas de una o varias distribuciones conjuntas. La metodología empleada aquí incluye, para el primer caso, densidades kernel de Epanechinikov y, para el segundo, cópulas multivariadas y algunas técnicas de la TVE. 5

4 Una de las razones que explica el irregular comportamiento de los histogramas es, sin duda, el pequeño tamaño de la muestra considerada (60 observaciones).

5 Para una muestra de observaciones $x_{1}, \ldots, x_{n}$ el estimador de densidad kernel está dado por la función $\hat{f}(x)=\frac{\sum_{j=1}^{n} K_{h}\left(x-x_{j}\right)}{n \int K_{h}(t-u) d t}$, donde $K_{h}(x)=\left(\frac{1}{h}\right) K\left(\frac{x}{h}\right), K(u)=\left(\frac{3}{4}\right)\left(1-u^{2}\right) 1_{\{|u|<1\}}$ es la función kernel de Epanechinikov y $h$ el ancho de banda. 


\section{IMPLEMENTACION DEL METODO DE CALCULO DEL CER}

\subsection{Distribuciones leptocurticas y cópulas}

Entre las razones más citadas para explicar la poca confiabilidad de los estimadores del $V_{a} R_{q}$ destaca, sin duda, la conocida leptocurtosis -o colas gordas- que afecta a las distribuciones de pérdidas como las de la Figura 2. La presencia de colas gordas en las distribuciones de créditos es ampliamente conocida y muchas de las explicaciones están centradas, entre otras cosas, en la alta correlación de los movimientos de las exposiciones, que echa por tierra los supuestos de normalidad implícitos en las medidas de valor en riesgo. ${ }^{6}$ La recomendación más usual para atacar este problema es utilizar algún método estocástico alternativo que permita capturar los diferentes comportamientos de las distribuciones marginales afectadas por colas gordas (Ramírez, 2004).

Las cópulas son, sin duda, uno de los recursos más socorridos por los analistas al momento de modelar la estructura de dependencia de esas distribuciones (Bouyé et al., 2000 y Embrechts et al., 2002). El motivo de su popularidad reside en la misma definición pues, como se sabe, se trata de una función que expresa una distribución conjunta de probabilidad como una función de distribuciones marginales. Y eso permite modelar dependencia entre distribuciones que, incluso, son completamente diferentes entre sí. En el caso de portafolios de créditos, el uso de cópulas es todavía más justificado porque los créditos son productos que dependen de eventos conjuntos (como podrían ser las obligaciones de deudas colateralizadas) que son mejor valuados con instrumentos estadísticos que correlacionan las colas de las distribuciones.

En la literatura financiera se han ensayado diversos tipos de cópulas para modelar la probabilidad de que dos mercados caigan en crisis al mismo tiempo pero sin emplear métodos comparativos que justifiquen la elección de la mejor distribución subyacente en cada cópula (Kole et al., 2005). Por esa razón, es conveniente proponer criterios de bondad de ajuste que hagan más objetiva la elección de las familias de cópulas y, de esa manera, evitar su uso indiscriminado y sin fundamento. El primer paso en esa dirección es comparar la capacidad predictiva de las cópulas sobre bases comunes, proponiendo los siguientes supuestos:

1) Las estimaciones conjuntas de los parámetros de riesgo $\left(\hat{T}_{i 1}, C \hat{C} F_{i 1}\right)_{i=1}^{M}, \ldots,\left(\hat{T} I_{i n}, C \hat{C} F_{i n}\right)_{i=1}^{M}$ son independientes e idénticamente distribuidas (i.i.d.) al momento de construir la cópula asociada del vector aleatorio $\left(P D_{i}, E A D_{i}\right)_{i=1}^{M}$.

6 Suponer normalidad multivariada en distribuciones de exposiciones leptocúrticas tiende a subestimar el valor en riesgo de un portafolio (véase Frey y McNeil, 2003; y Hult y Lindskog, 2001). 
2) Las estimaciones conjuntas $\left(L \hat{G} D_{p 1}\right)_{p=1}^{K}, \ldots,\left(L \hat{G} D_{p m}\right)_{p=1}^{K}$ son i.i.d en la determinación de la cópula asociada del vector aleatorio $\left(L G D_{p}\right)_{p=1}^{K}$.

3) La correlación serial ocasionada por el traslape de ventanas de observación es despreciable.

4) Los parámetros de riesgo $P D$ y $C C F$ son dependientes entre sí y entre cubetas y su estructura de dependencia está dada por la cópula $C^{{ }^{P}}{ }^{P D E D}$. Más precisamente: se supone que el vector aleatorio $2 k$-dimensional

$$
{ }_{p} P D E A D=\left(C C F_{i 1}, T I_{i 1}, \ldots, C C F_{i k}, T I_{i k}\right)^{\prime}
$$

tiene marginales absolutamente continuas, donde $\left\{i_{1}, \ldots, i_{k}\right\} \subset\{1, \ldots, M\}$ es un conjunto de cubetas de $\operatorname{los} p=1, \ldots, K$ productos independientes entre sí.

5) A nivel de agregación por productos, el vector aleatorio $K$-dimensional

$$
L G D=\left(L G D_{1}, \ldots, L G D_{K}\right)^{\prime}
$$

está definido por marginales absolutamente continuas y alguna cópula asociada $C^{L G D}$.

6) El vector aleatorio $\left(L G D_{p}\right)_{p=1}^{K}$ es independiente de los parámetros de riesgo $\left(P D_{i}, E A D_{i}\right)_{i=1}^{M}$.

\subsection{Comparación entre ajustes de cópulas}

El segundo paso consiste en adoptar una estrategia de comparación gradual. Es decir, hacer uso, primero, de las estructuras más simples, como las de independencia y gaussiana y, luego, comparar sus resultados con el de otras familias de cópulas más complejas que incorporan efectos conjuntos de dependencia extrema. La idea es ir descartando pormenorizadamente las razones por las que las cópulas ofrecen mejores ajustes o tienen mayor capacidad predictiva que otras. Para tal efecto empezaremos por ajustar el primer tipo de cópulas a los datos de los parámetros de riesgo $P D$ y $C C F$, suponiendo que $L G D$ es constante e igual a su media histórica y que el estimador de la matriz de correlaciones de la cópula gaussiana es (ver Frey, 2003):

$$
\hat{\Sigma}=\left(\sum_{k=1}^{n} y_{k} y_{k}{ }^{\prime}\right)_{i j}
$$


donde $y_{k}=\left(\Phi^{-1}\left(\hat{F}_{1}\left(x_{i k}\right)\right), \ldots, \Phi^{-1}\left(\hat{F}_{d}\left(x_{d k}\right)\right)\right)$ ' y $\hat{F}_{i}$ es la función de distribución empírica del vector de observaciones $\left(x_{i 1}, \ldots, x_{i n}\right)^{\prime}$ de la variable aleatoria $X_{i}$, para cada $i=1, \ldots, d$. Suponer a $L G D$ como constante es sólo un recurso heurístico para considerar las condiciones más elementales sobre las que se estiman las medidas de riesgo con las cópulas gaussiana y de independencia. El relajamiento posterior de este supuesto permitirá observar, por comparación, su importancia en la estimación del riesgo. En todos los ajustes marginales el ancho de banda es fijado por la regla de Silverman. ${ }^{7}$

Los ajustes resultantes de aplicar las cópulas de independencia y gaussiana (ver Tablas 4 y 5) no presentan mejora alguna respecto de su benchmark o método histórico, ya que no obstante que los nuevos valores de $P E$ muestran poca sensibilidad a los dos modelos de estructura de dependencia, las simulaciones empíricas de $V_{a} R_{q}$ (columnas VaRqEmp de la Tabla 3) son ostensiblemente superiores. Esto quiere decir que las distribuciones de pérdidas simuladas con los ajustes de las cópulas de independencia y gaussiana adolecen aún de mayores problemas que su benchmark al subestimar las colas de las distribuciones de pérdidas subyacentes en cada producto y en el portafolio total. Efectivamente, como se destaca en el último renglón de las Tablas 4 y 5 , los valores de $\mathrm{VaR}_{q}$ son relativamente menores con las cópulas de independencia y gaussiana $(4,27 \%$ y $5,04 \%$, respectivamente) que con el método histórico $(5,21 \%)$ para un nivel del $99 \%$ de confianza.

Una sugerencia para corregir la subestimación en las distribuciones de pérdidas afectadas por leptocurtosis es tratar con cópulas cuyas marginales regulen la pesadez de las colas como, en efecto, sucede con las familias $t$ de Student (Ramírez, 2004). De entre las más socorridas por su flexibilidad y buen desempeño en aplicaciones de medición de riesgos destacan las cópulas $t$ de Student simétricas (ver Embrechts et al., 2002). ${ }^{8}$ De acuerdo con la Tabla 6, la aplicación de esta cópula mejora las estimaciones del $\mathrm{VaR}_{q}$ de los dos ajustes anteriores al arrojar resultados más cercanos a los obtenidos por el método histórico. Así tenemos, por ejemplo que para un nivel de confianza del 99\%, el cuantil de la distribución de pérdidas del portafolio total es de $5,33 \%$, esto es: una cifra apenas superior a su contraparte histórica de la Tabla 3.

7 Esta regla permite determinar un ancho de banda $h$ óptimo (para ajustar densidades normales) mediante la fórmula $(40 \sqrt{\pi} / n)^{1 / 5} \hat{\sigma}$, donde $n$ es el número de observaciones y $\hat{\sigma}$ la volatilidad estimada.

$8 \quad \mathrm{Al}$ ajustar esta cópula se asumen los mismos supuestos sobre LGD y las densidades kernel. La matriz de correlaciones se estima con la ecuación (7) tomando $y_{k}=\left(t_{\alpha}{ }^{-1}\left(\hat{F}_{1}\left(x_{i k}\right)\right), \ldots, t_{\alpha}{ }^{-1}\left(\hat{F}_{d}\left(x_{d k}\right)\right)\right)^{\prime}$, donde $t_{\alpha}$ denota la distribución univariada de Student cuyo parámetro de grados de libertad $\alpha$ es estimado con el algoritmo 1 del Apéndice. 


\section{TABLA 4}

ESTIMACIONES DE $P E \mathrm{Y} V a R_{q}$ AJUSTANDO LA COPULA DE INDEPENDENCIA A $P D$ Y $C C F$ DEL PORTAFOLIO

\begin{tabular}{|c|r|r|r|r|r|}
\hline \multirow{2}{*}{ Producto } & \multirow{2}{*}{$\mathrm{PE}$} & \multicolumn{4}{|c|}{$\mathrm{VaR}_{\mathrm{q}} \mathrm{Emp}$} \\
\cline { 3 - 6 } & & $99 \%$ & $99,5 \%$ & $99,9 \%$ & $99,95 \%$ \\
\hline $\mathrm{A}$ & $8,91 \%$ & $10,61 \%$ & $10,79 \%$ & $11,20 \%$ & $11,32 \%$ \\
$\mathrm{~B}$ & $5,37 \%$ & $6,58 \%$ & $6,67 \%$ & $6,96 \%$ & $7,01 \%$ \\
$\mathrm{C}$ & $0,38 \%$ & $1,05 \%$ & $1,15 \%$ & $1,31 \%$ & $1,35 \%$ \\
$\mathrm{D}$ & $1,36 \%$ & $1,83 \%$ & $1,88 \%$ & $2,00 \%$ & $2,04 \%$ \\
$\mathrm{E}$ & $7,36 \%$ & $11,48 \%$ & $11,87 \%$ & $12,65 \%$ & $12,80 \%$ \\
$\mathrm{~F}$ & $8,88 \%$ & $10,76 \%$ & $10,99 \%$ & $11,37 \%$ & $11,61 \%$ \\
$\mathrm{G}$ & $9,73 \%$ & $11,72 \%$ & $11,93 \%$ & $12,42 \%$ & $12,65 \%$ \\
$\mathrm{H}$ & $6,00 \%$ & $7,65 \%$ & $7,74 \%$ & $7,89 \%$ & $7,96 \%$ \\
$\mathrm{I}$ & $1,73 \%$ & $2,30 \%$ & $2,35 \%$ & $2,46 \%$ & $2,51 \%$ \\
$\mathrm{~J}$ & $2,87 \%$ & $4,39 \%$ & $4,52 \%$ & $4,84 \%$ & $4,93 \%$ \\
\hline Total & $3,83 \%$ & $4,27 \%$ & $4,33 \%$ & $4,43 \%$ & $4,47 \%$ \\
\hline
\end{tabular}

TABLA 5

ESTIMACIONES DE $P E Y V R_{q}$ AJUSTANDO UNA COPULA GAUSSIANA A PD Y CCF DEL PORTAFOLIO

\begin{tabular}{|c|c|r|r|r|r|}
\hline \multirow{2}{*}{ Producto } & \multirow{2}{*}{$\mathrm{PE}$} & \multicolumn{4}{|c|}{$\mathrm{VaR}_{\mathrm{q}} \mathrm{Emp}$} \\
\cline { 3 - 6 } & & $99 \%$ & $99,5 \%$ & $99,9 \%$ & $99,95 \%$ \\
\hline $\mathrm{A}$ & $8,92 \%$ & $10,33 \%$ & $10,52 \%$ & $10,85 \%$ & $10,93 \%$ \\
$\mathrm{~B}$ & $5,36 \%$ & $6,82 \%$ & $6,96 \%$ & $7,17 \%$ & $7,21 \%$ \\
$\mathrm{C}$ & $0,37 \%$ & $0,94 \%$ & $1,02 \%$ & $1,14 \%$ & $1,21 \%$ \\
$\mathrm{D}$ & $1,37 \%$ & $2,41 \%$ & $2,51 \%$ & $2,68 \%$ & $2,72 \%$ \\
$\mathrm{E}$ & $7,41 \%$ & $12,25 \%$ & $12,66 \%$ & $13,55 \%$ & $14,01 \%$ \\
$\mathrm{~F}$ & $8,94 \%$ & $12,07 \%$ & $12,41 \%$ & $13,11 \%$ & $13,19 \%$ \\
$\mathrm{G}$ & $9,74 \%$ & $13,52 \%$ & $13,83 \%$ & $14,55 \%$ & $14,73 \%$ \\
$\mathrm{H}$ & $6,00 \%$ & $7,43 \%$ & $7,51 \%$ & $7,72 \%$ & $7,74 \%$ \\
$\mathrm{I}$ & $1,72 \%$ & $2,19 \%$ & $2,24 \%$ & $2,34 \%$ & $2,36 \%$ \\
$\mathrm{~J}$ & $2,88 \%$ & $4,60 \%$ & $4,69 \%$ & $4,86 \%$ & $4,93 \%$ \\
\hline Total & $3,84 \%$ & $5,04 \%$ & $5,17 \%$ & $5,39 \%$ & $5,42 \%$ \\
\hline
\end{tabular}


TABLA 6

ESTIMACIONES DE $P E$ Y $V_{a} R_{q}$ AJUSTANDO UNA COPULA T DE STUDENT SIMETRICA A $P D$ Y CCF DE TODO EL PORTAFOLIO

\begin{tabular}{|c|c|r|r|r|r|}
\hline \multirow{2}{*}{ Producto } & \multirow{2}{*}{ PE } & \multicolumn{4}{|c|}{ VaR $_{\mathrm{q}}$ Emp } \\
\cline { 3 - 6 } & & \multicolumn{1}{|c|}{$99 \%$} & $99,5 \%$ & $99,9 \%$ & $99,95 \%$ \\
\hline $\mathrm{A}$ & $8,90 \%$ & $10,94 \%$ & $11,12 \%$ & $11,49 \%$ & $11,73 \%$ \\
$\mathrm{~B}$ & $5,36 \%$ & $7,19 \%$ & $7,32 \%$ & $7,61 \%$ & $7,67 \%$ \\
$\mathrm{C}$ & $0,37 \%$ & $0,98 \%$ & $1,08 \%$ & $1,21 \%$ & $1,25 \%$ \\
$\mathrm{D}$ & $1,36 \%$ & $2,56 \%$ & $2,69 \%$ & $2,84 \%$ & $2,87 \%$ \\
$\mathrm{E}$ & $7,37 \%$ & $12,77 \%$ & $13,35 \%$ & $14,07 \%$ & $14,42 \%$ \\
$\mathrm{~F}$ & $8,90 \%$ & $12,62 \%$ & $13,05 \%$ & $13,66 \%$ & $13,93 \%$ \\
$\mathrm{G}$ & $9,70 \%$ & $14,18 \%$ & $14,49 \%$ & $15,30 \%$ & $15,47 \%$ \\
$\mathrm{H}$ & $6,00 \%$ & $7,80 \%$ & $7,91 \%$ & $8,13 \%$ & $8,17 \%$ \\
$\mathrm{I}$ & $1,72 \%$ & $2,27 \%$ & $2,34 \%$ & $2,46 \%$ & $2,48 \%$ \\
$\mathrm{~J}$ & $2,87 \%$ & $4,79 \%$ & $4,91 \%$ & $5,08 \%$ & $5,14 \%$ \\
\hline Total & $3,82 \%$ & $5,33 \%$ & $5,46 \%$ & $5,68 \%$ & $5,78 \%$ \\
\hline
\end{tabular}

Las dificultades encontradas en las estimaciones anteriores del $\mathrm{VaR}_{q}$ no quedan, sin embargo, completamente superadas con la introducción de la cópula $t$ de Student simétrica. Falta, todavía, determinar los efectos de la asimetría en la estructura de dependencia de los parámetros de riesgo y ponderar los efectos de la constancia de LGD. ${ }^{9}$ El primer problema es regularmente enfrentado con el uso de las cópulas elípticas generalizadas y el segundo con el de las cópulas agrupadas (Frahm y Junker, 2003).

Los algoritmos utilizados para estimar las cópulas elípticas y agrupadas son, básicamente, no paramétricos y están basados en la correlación de rangos y los coeficientes de dependencia de la cola. Las justificaciones para utilizarlos aquí residen en que: 1) a diferencia de su versión lineal, las correlaciones de rango proveen medidas robustas de dependencia entre variables aleatorias invariantes bajo transformaciones estrictamente crecientes que son derivadas directamente de las cópulas (ver Embrechts et al., 2002) y 2) el concepto de dependencia de la cola captura los efectos del fenómeno de valores extremos conjuntos entre los parámetros de riesgo que, de otra forma sería imposible estimar. Los procedimientos para su implementación están basados en los trabajos de Kostadinov (2005) y Frahm y Junker (2003) quienes proporcionan, respectivamente, métodos para estimar los parámetros de cópulas elípticas simétricas y generalizadas. En el Apéndice A3 se presenta el algoritmo usado para estimar los parámetros de cópulas agrupadas, que no es más que una adaptación de ambos métodos a las versiones $t$ de Student de las familias de cópulas arriba mencionadas.

9 Frahm (2003) encuentra que la presencia de asimetría es importante en la modelación de las estructuras de dependencia de los factores de riesgo de mercado. No encontramos, sin embargo, evidencia de la aplicación de la asimetría para el caso de riesgo de crédito. 
En lo que toca a las cópulas elípticas generalizadas cabe destacar que el procedimiento de modelación de la estructura de dependencia de los vectores aleatorios ${ }_{p}$ PDEAD y LGD requiere, inicialmente, de ajustar las cópulas $C^{p^{\text {PDEAD }}}$ y $C^{\mathbf{L G D}}$ a cada producto $p$, tal como se especifica en el algoritmo 2 del Apéndice A3. Luego, es necesario asociar densidades kernel de suavización a las observaciones históricas de $P D, E A D$ y $L G D$ para construir las distribuciones marginales de los parámetros de riesgo contenidos en los vectores anteriores. Finalmente hay que definir las pérdidas (como porcentaje de su saldo) para cada cubeta $i$ como:

$$
L_{i}=T I_{i} * C C F_{i} * L G D_{i}
$$

y simular sus series $L_{i 1}, \ldots, L_{i N}$, para cada cubeta $i \in\left\{i_{1}, \ldots, i_{k}\right\}$ del producto $p$ con base en las estimaciones ${ }_{p} P D \hat{E} A D_{1}, \ldots,{ }_{p} P D \hat{E} A D_{N}, p=1, \ldots, K$ y $L \hat{G} D_{1}, \ldots, L \hat{G} D_{N}$ de los parámetros de riesgo. En la agregación de las pérdidas por producto hay que ponderar el saldo $e_{i}$ de cada cubeta $i$ por el de cada producto $p$ conforme a la siguiente fórmula:

$$
L_{p j}=\sum_{i \in\left\{i_{1}, \ldots, i_{k}\right\}} \frac{e_{i}}{\sum_{j \in\left\{i_{1}, \ldots, i_{k}\right\}^{e}} e_{j}} L_{i j}, \quad j=1, \ldots, N
$$

Como paso previo y con el objeto de evaluar la asimetría en la estructura de dependencia de los parámetros de riesgo se emplea el algoritmo 1 del Apéndice A3 para estimar los índices de dependencia extrema (denotado con el parámetro $\alpha$ ) de cada producto y no sólo del portafolio total, como en efecto acontece con los anteriores ajustes. Los resultados de la Tabla 7 muestran que los índices obtenidos para los parámetros $P D$ y $C C F$ son bastante diferentes entre productos. Cabe mencionar que este índice mide la ocurrencia de observaciones conjuntas extremas de tal suerte que un menor valor de $\alpha$ implica un mayor grado de dependencia entre las observaciones extremas conjuntas. En particular los productos $\mathrm{H}$ y $\mathrm{J}$ presentan los menores índices de la cola o, dicho de otra manera, los mayores niveles de dependencia conjunta extrema, mientras que el portafolio exhibe la situación totalmente contraria, al tiempo que los productos C, D, F y G presentan un grado de dependencia extrema similar.

Las nuevas corridas de los valores agregados de $P E$ y de $V_{a} R_{q}$ que resultan de ajustar las cópulas t simétrica y generalizada a cada producto e índice de cola $\alpha$ aparecen desplegados en las Tablas 8 y 9. Los valores de PE se calculan como el promedio aritmético de las observaciones obtenidas por una simulación Montecarlo mientras que los estimadores de $\operatorname{VaR}_{q}$ son obtenidos por los métodos POT $^{10}$ (Columna VaRqPOT), Empírico (columna VaRqEmp) y distribución Beta (VaRqBeta). ${ }^{11}$ En el Apéndice A1 se describe la implementación del cálculo de los estimadores mencionados.

10 Para revisar los resultados más importantes de este método derivado de la TVE véase Embrechts et al. (1997).

11 Con excepción del método POT, los otros dos son ampliamente utilizados en los análisis de riesgo de crédito. Así que una comparación entre ellos es una buena oportunidad para comprobar las bondades de dicho método para el caso particular de portafolios de créditos al menudeo. 
TABLA 7

ESTIMACIONES DEL PARAMETRO DE DEPENDENCIA EXTREMA POR PRODUCTO Y PORTAFOLIO TOTAL

\begin{tabular}{|c|c|c|}
\hline Producto & $\begin{array}{c}\text { Parámetro } \alpha_{p} \\
\text { (dependencia extrema) }\end{array}$ & Error estándar \\
\hline A & 20,5 & $(1,38)$ \\
B & 38,8 & $(1,47)$ \\
C & 11,8 & $(0,88)$ \\
D & 14,2 & $(1,13)$ \\
E & 67,9 & $(1,51)$ \\
F & 9,7 & $(0,73)$ \\
H & 10,8 & $(0,67)$ \\
I & 2,0 & $(0,15)$ \\
J & 22,1 & $(1,41)$ \\
Total & 3,2 & $(0,19)$ \\
\hline
\end{tabular}

Al comparar los ajustes de las dos cópulas por producto y nivel de confianza observamos que las estimaciones del $\mathrm{VaR}_{q}$ son bastante parecidas en ambos casos o, dicho de otro modo, que las especificaciones de asimetría dadas por las cópulas elípticas generalizadas (Tabla 9) tienen un impacto casi nulo en la modelación de la estructura de dependencia de $P D$ y $C C F$. Pero esto no quiere decir que el procedimiento haya sido estéril. Al contrario, el cálculo de los índices de la cola $\alpha$ por producto mejora sensiblemente las estimaciones empíricas de $\mathrm{VaR}_{q}$ de las dos últimas tablas con relación a la 6 . El análisis de la dependencia conjunta extrema por producto permite, de hecho, capturar efectos particulares sobre las medidas de riesgo que pasan inadvertidos cuando se utiliza un solo índice de cola para todo el portafolio.

Para profundizar más en este último punto introduciremos como instrumento de comparación a la cópula $t$ de Student agrupada que se distingue no sólo por su capacidad de relacionar grupos de variables aleatorias sino, fundamentalmente, por mantener la diferenciación de eventos extremos entre grupos. La aplicación de esta cópula a los parámetros de riesgo $P D$ y $C C F$ del portafolio total incorpora la matriz de correlaciones $P$ y los estimadores de los parámetros con $\alpha_{p}$ grados de libertad para cada producto $p$ de acuerdo con el paso (i) del algoritmo 2 (véase Apéndice A3). Los resultados de la agregación de las pérdidas simuladas $\left({ }_{p} P D E A D\right)_{p=1}^{K}$ por producto y portafolio total se exhiben en la Figura 3 donde se muestran los histogramas de las distribuciones de pérdidas.

Al comparar la forma de los histogramas de las Figuras 2 y 3 resalta, al menos visualmente, la mayor área en la cola derecha de las distribuciones de pérdidas producida por las cópulas que modelan la dependencia conjunta extrema; lo cual quiere decir que con este tipo de cópulas es posible observar pérdidas superiores que son 


\begin{tabular}{|c|c|c|c|c|c|c|c|c|c|c|c|}
\hline \multirow{14}{*}{ 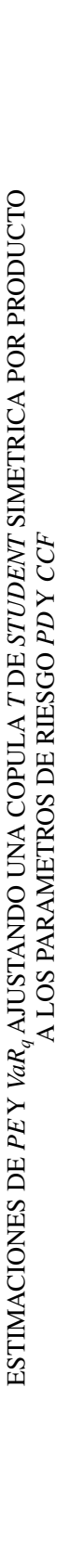 } & $\begin{array}{l}\stackrel{2}{2} \\
2 \\
\sigma\end{array}$ & $\begin{array}{l}\stackrel{0}{\stackrel{2}{a}} \\
=\end{array}$ & $\begin{array}{l}\stackrel{0}{2} \\
\text { mे } \\
\infty\end{array}$ & $\stackrel{\circ}{0}$ & $\begin{array}{l}\stackrel{0}{0} \\
\infty \\
i\end{array}$ & $\begin{array}{l}0 \\
0 \\
0 \\
2 \\
\pm \\
\pm\end{array}$ & $\begin{array}{l}00 \\
0 \\
\pm\end{array}$ & $\begin{array}{l}\stackrel{0}{2} \\
\stackrel{2}{ \pm} \\
\dot{J}\end{array}$ & $\begin{array}{l}\overbrace{0}^{0} \\
\text { ते } \\
\infty\end{array}$ & $\begin{array}{l}00 \\
n \\
n \\
i\end{array}$ & 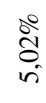 \\
\hline & $\begin{array}{l}\stackrel{2}{\sigma} \\
\text { बे }\end{array}$ & $\begin{array}{l}0_{0}^{\circ} \\
\stackrel{n}{=} \\
=\end{array}$ & $\begin{array}{l}\infty^{0} \\
\frac{\infty}{\infty}\end{array}$ & $\begin{array}{l}0 \\
\infty \\
0 \\
0\end{array}$ & $\begin{array}{l}\stackrel{0}{0} \\
\text { i } \\
\text { i }\end{array}$ & $\begin{array}{l}\stackrel{0}{0} \\
\infty \\
\infty \\
2\end{array}$ & $\begin{array}{l}\stackrel{\circ}{N} \\
\stackrel{ \pm}{ \pm}\end{array}$ & $\begin{array}{l}\stackrel{0}{0} \\
\infty \\
\stackrel{2}{ \pm}\end{array}$ & $\begin{array}{l}0 \\
8 \\
\infty\end{array}$ & $\begin{array}{l}\stackrel{0}{a} \\
\stackrel{a}{+} \\
i\end{array}$ & $\begin{array}{l}\bigotimes^{\circ} \\
\infty \\
+\end{array}$ \\
\hline & 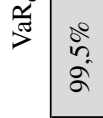 & $\begin{array}{l}\stackrel{0}{\infty} \\
0 \\
=\end{array}$ & $\begin{array}{l}00 \\
i 0 \\
i \\
i\end{array}$ & $\begin{array}{l}0 \\
\vdots \\
\infty \\
0\end{array}$ & $\begin{array}{l}\stackrel{\oplus}{\tilde{\sigma}} \\
\stackrel{i}{i}\end{array}$ & 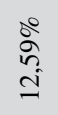 & $\begin{array}{l}\stackrel{0}{+} \\
\stackrel{+}{2} \\
\end{array}$ & $\begin{array}{l}80 \\
\stackrel{0}{0} \\
\stackrel{2}{2}\end{array}$ & $\begin{array}{l}\stackrel{\circ}{\circ} \\
\text { के }\end{array}$ & $\begin{array}{l}\text { o̊ } \\
\text { in } \\
\text { i }\end{array}$ & $\begin{array}{l}\stackrel{0}{0} \\
\stackrel{\infty}{+} \\
\dot{f}\end{array}$ \\
\hline & $\begin{array}{l}\stackrel{2}{a} \\
\text { a }\end{array}$ & $\begin{array}{l}\stackrel{0}{0} \\
\infty \\
0 \\
0\end{array}$ & 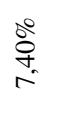 & $\frac{\circ}{2}$ & $\begin{array}{l}\stackrel{0}{0} \\
\text { in } \\
i\end{array}$ & $\begin{array}{l}80 \\
8 \\
0 \\
0\end{array}$ & 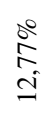 & $\begin{array}{l}\stackrel{\circ}{N} \\
\text { ஸे }\end{array}$ & $\frac{0}{n}$ & 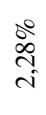 & $\begin{array}{l}0 \\
0 \\
0 \\
+\end{array}$ \\
\hline & \multirow{4}{*}{ 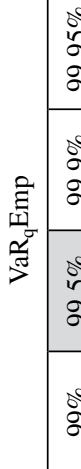 } & $\begin{array}{l}\stackrel{0}{0} \\
\infty \\
=\end{array}$ & $\begin{array}{l}b^{0} \\
\text { b } \\
r\end{array}$ & 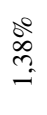 & $\begin{array}{l}\stackrel{0}{0} \\
\stackrel{0}{0} \\
\text { î }\end{array}$ & 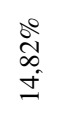 & $\begin{array}{l}\delta 0 \\
8 \\
\dot{ \pm} \\
\pm\end{array}$ & $\begin{array}{l}\frac{0}{2} \\
n \\
n\end{array}$ & $\begin{array}{l}b_{0}^{\circ} \\
\stackrel{1}{\infty} \\
\infty\end{array}$ & $\begin{array}{l}\stackrel{0}{a} \\
\stackrel{a}{i} \\
\text { i }\end{array}$ & 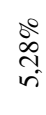 \\
\hline & & $\begin{array}{l}\stackrel{0}{\infty} \\
\stackrel{0}{n} \\
=\end{array}$ & $\begin{array}{l}\frac{8}{2} \\
\text { ñ }\end{array}$ & $\stackrel{\text { mे }}{2}$ & $\begin{array}{l}\stackrel{0}{8} \\
\text { aे } \\
\text { i }\end{array}$ & 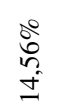 & $\begin{array}{l}\infty \\
\infty \\
\infty \\
\infty\end{array}$ & $\begin{array}{l}\frac{0}{m} \\
\stackrel{n}{n}\end{array}$ & $\begin{array}{l}\vdots \\
\text { ஸे } \\
\infty\end{array}$ & $\begin{array}{l}\stackrel{0}{i} \\
\stackrel{n}{i} \\
i\end{array}$ & $\begin{array}{l}\stackrel{0}{N} \\
\text { in }\end{array}$ \\
\hline & & $\begin{array}{l}\stackrel{\circ}{\Xi} \\
=\end{array}$ & 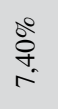 & $\stackrel{0}{=}$ & $\begin{array}{l}\frac{0}{2} \\
\text { io } \\
\text { i }\end{array}$ & $\begin{array}{l}\stackrel{0}{a} \\
a+ \\
\text { ma }\end{array}$ & $\frac{\stackrel{\circ}{2}}{2}$ & 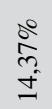 & $\begin{array}{l}0 \\
8 \\
\infty\end{array}$ & 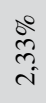 & 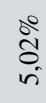 \\
\hline & & $\begin{array}{l}\frac{0}{\sigma} \\
\frac{0}{2}\end{array}$ & $\begin{array}{l}\stackrel{0}{0} \\
\stackrel{1}{r} \\
i\end{array}$ & $\stackrel{8}{0}$ & $\begin{array}{l}00 \\
0 \\
n \\
i \\
i\end{array}$ & $\begin{array}{l}\stackrel{0}{8} \\
8 \\
\stackrel{0}{2}\end{array}$ & $\begin{array}{l}\stackrel{0}{\infty} \\
\stackrel{0}{a} \\
\text { I }\end{array}$ & $\begin{array}{l}80 \\
\infty \\
\infty \\
\end{array}$ & $\begin{array}{l}\stackrel{0}{0} \\
\infty \\
i\end{array}$ & $\begin{array}{l}0 \\
\infty \\
i \\
i\end{array}$ & $\begin{array}{l}\stackrel{0}{\circ} \\
\stackrel{+}{+}\end{array}$ \\
\hline & \multirow{4}{*}{$\begin{array}{l}5 \\
0 \\
\alpha^{5} \\
\frac{\alpha}{\sigma} \\
=\end{array}$} & 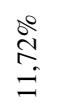 & 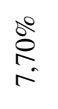 & 兽 & $\begin{array}{l}\stackrel{0}{2} \\
\text { aे } \\
\text { in }\end{array}$ & $\begin{array}{l}\stackrel{0}{0} \\
2 \\
\vdots \\
\dot{J}\end{array}$ & $\begin{array}{l}\stackrel{\circ}{\Xi} \\
\stackrel{ \pm}{ \pm}\end{array}$ & 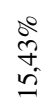 & $\begin{array}{l}\stackrel{0}{0} \\
\ddot{\infty}\end{array}$ & $\begin{array}{l}\stackrel{0}{a} \\
\stackrel{a}{i}\end{array}$ & $\begin{array}{l}\frac{0}{\pi} \\
\text { in }\end{array}$ \\
\hline & & $\begin{array}{l}\stackrel{\circ}{n} \\
= \\
=\end{array}$ & 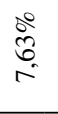 & 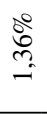 & $\begin{array}{l}\stackrel{0}{2} \\
\infty \\
i\end{array}$ & 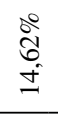 & 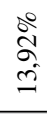 & $\frac{\stackrel{2}{=}}{\stackrel{0}{n}}$ & $\begin{array}{l}\stackrel{0}{0} \\
\text { त) } \\
\infty\end{array}$ & $\begin{array}{l}\stackrel{0}{0} \\
\stackrel{n}{n} \\
i\end{array}$ & 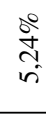 \\
\hline & & $\begin{array}{l}\stackrel{\circ}{i n} \\
=\end{array}$ & $\underset{\frac{6}{\sigma}}{\stackrel{5}{r}}$ & $\stackrel{\stackrel{0}{\leftrightarrows}}{=}$ & $\begin{array}{l}\stackrel{0}{0} \\
\infty \\
0 \\
i\end{array}$ & $\begin{array}{l}00 \\
\infty \\
i n \\
2\end{array}$ & $\frac{\stackrel{8}{ \pm}}{2}$ & 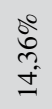 & $\begin{array}{c}\stackrel{0}{\infty} \\
\infty\end{array}$ & 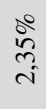 & $\begin{array}{l}80 \\
8 \\
\text { in }\end{array}$ \\
\hline & & $\begin{array}{l}\stackrel{\circ}{\sigma} \\
\stackrel{0}{0}\end{array}$ & 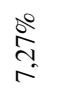 & $\stackrel{0}{0}$ & 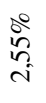 & $\begin{array}{l}00 \\
\infty \\
0 \\
\text { తి }\end{array}$ & $\begin{array}{l}\stackrel{a}{2} \\
\text { in }\end{array}$ & $\begin{array}{l}\stackrel{0}{0} \\
\circ \\
\text { ले }\end{array}$ & $\frac{2}{a}$ & $\begin{array}{l}\stackrel{8}{2} \\
\text { ते }\end{array}$ & $\begin{array}{l}\stackrel{0}{\sigma} \\
\text { ๙े }\end{array}$ \\
\hline & $\underline{\underline{2}}$ & $\begin{array}{l}\infty \\
\infty \\
\infty \\
\infty\end{array}$ & $\begin{array}{l}\stackrel{0}{m} \\
\text { ñ } \\
\text { nे }\end{array}$ & 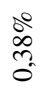 & 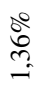 & $\begin{array}{l}\stackrel{\circ}{2} \\
\text { ले }\end{array}$ & 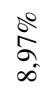 & $\begin{array}{l}\circ \\
\hat{n} \\
a\end{array}$ & $\begin{array}{l}8 \\
8 \\
0\end{array}$ & $\stackrel{\stackrel{ }{N}}{\approx}$ & $\begin{array}{l}0 \\
\infty \\
\infty \\
i\end{array}$ \\
\hline & $\begin{array}{l}\frac{0}{0} \\
\frac{0}{0} \\
0 \\
0\end{array}$ & $\varangle$ & 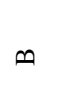 & $U$ & $\theta$ & 피 & IL & ט & $I$ & - & $\sim$ \\
\hline
\end{tabular}




\begin{tabular}{|c|c|c|c|c|c|c|c|c|c|c|c|c|}
\hline \multirow{14}{*}{ 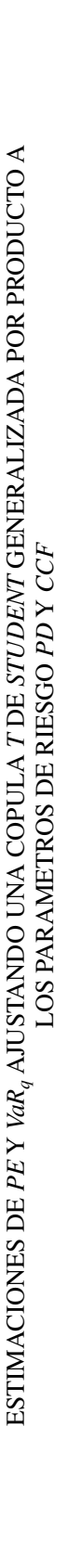 } & \multirow{4}{*}{ 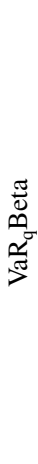 } & $\begin{array}{l}\stackrel{2}{2} \\
2 \\
2\end{array}$ & $\stackrel{\stackrel{\circ}{\rightleftharpoons}}{=}$ & $\begin{array}{l}\infty \\
\infty \\
m \\
\infty \\
\infty\end{array}$ & $\underset{-}{\stackrel{0}{0}}$ & $\begin{array}{l}\frac{0}{\infty} \\
0 \\
i\end{array}$ & 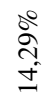 & 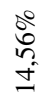 & $\begin{array}{l}\stackrel{0}{+} \\
\dot{\Delta} \\
\dot{ \pm}\end{array}$ & $\begin{array}{l}\stackrel{0}{0} \\
\stackrel{\text { N }}{\infty}\end{array}$ & $\begin{array}{l}\delta^{\circ} \\
i n \\
i n\end{array}$ & $\begin{array}{l}\stackrel{0}{0} \\
\text { ठै. } \\
\text { î }\end{array}$ \\
\hline & & $\begin{array}{l}\stackrel{2}{\alpha} \\
\text { à }\end{array}$ & 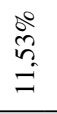 & $\frac{\stackrel{2}{I}}{\infty}$ & $\begin{array}{l}D^{0} \\
\stackrel{0}{0} \\
0\end{array}$ & $\begin{array}{l}\stackrel{0}{0} \\
\text { i } \\
\text { in }\end{array}$ & $\begin{array}{l}\stackrel{0}{\infty} \\
\stackrel{\infty}{\sigma} \\
\stackrel{0}{2}\end{array}$ & $\begin{array}{l}\frac{\partial}{I} \\
\pm \\
\pm\end{array}$ & $\begin{array}{l}\frac{8}{D} \\
\hat{n} \\
\pm \\
\pm\end{array}$ & $\begin{array}{l}\stackrel{0}{0} \\
2 \\
\infty\end{array}$ & $\begin{array}{l}\stackrel{\circ}{a} \\
\stackrel{+}{i} \\
\stackrel{0}{1}\end{array}$ & $\begin{array}{l}\stackrel{0}{ } \\
\stackrel{0}{\circ} \\
+\end{array}$ \\
\hline & & $\begin{array}{l}\stackrel{0}{2} \\
\text { aे }\end{array}$ & $\begin{array}{l}\stackrel{0}{0} \\
\text { है } \\
=\end{array}$ & $\begin{array}{l}\stackrel{\circ}{0} \\
\stackrel{0}{r}\end{array}$ & $\begin{array}{l}D^{\circ} \\
\dot{n}^{2} \\
0\end{array}$ & $\begin{array}{l}\stackrel{0}{\tilde{m}} \\
\stackrel{i}{i}\end{array}$ & $\begin{array}{l}\text { o̊ } \\
\stackrel{+}{2} \\
\text { in }\end{array}$ & $\begin{array}{l}\stackrel{\circ}{a} \\
\stackrel{2}{g}\end{array}$ & $\begin{array}{l}\stackrel{0}{\approx} \\
\tilde{n} \\
\end{array}$ & $\begin{array}{l}\delta_{0}^{0} \\
\infty \\
0 \\
\sim\end{array}$ & $\begin{array}{l}\text { o̊ } \\
\text { nn } \\
\text { in }\end{array}$ & $\begin{array}{l}\stackrel{0}{\infty} \\
\stackrel{+}{+} \\
+\end{array}$ \\
\hline & & $\begin{array}{l}\stackrel{2}{ } \\
\text { aे }\end{array}$ & $\begin{array}{l}\stackrel{0}{0} \\
\text { 心o } \\
0 \\
0\end{array}$ & مे & $\frac{80}{\circ}$ & $\begin{array}{l}\stackrel{0}{0} \\
\text { in } \\
i\end{array}$ & $\begin{array}{l}\text { ठ̊ } \\
\stackrel{0}{0} \\
=\end{array}$ & $\begin{array}{l}\stackrel{\circ}{+} \\
\underset{j}{i}\end{array}$ & $\begin{array}{l}\frac{b}{\pi} \\
\stackrel{2}{2}\end{array}$ & $\frac{b^{\circ}}{n}$ & $\begin{array}{l}\delta_{0}^{0} \\
\text { N } \\
i\end{array}$ & $\begin{array}{l}\stackrel{0}{0} \\
\stackrel{m}{f}\end{array}$ \\
\hline & \multirow{4}{*}{ 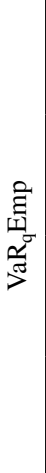 } & $\begin{array}{l}\stackrel{0}{2} \\
\stackrel{2}{\sigma} \\
\sigma\end{array}$ & $\begin{array}{l}\text { 号 } \\
\text { à } \\
\text { ปn }\end{array}$ & $\begin{array}{l}\stackrel{\circ}{\circ} \\
\text { ò }\end{array}$ & 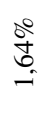 & $\begin{array}{l}\stackrel{0}{2} \\
\text { ลे }\end{array}$ & $\begin{array}{l}0 \\
\infty \\
0 \\
0 \\
0\end{array}$ & $\begin{array}{l}\stackrel{0}{0} \\
\dot{m} \\
\stackrel{2}{ \pm}\end{array}$ & $\begin{array}{l}\stackrel{\circ}{\circ} \\
\stackrel{2}{n}\end{array}$ & 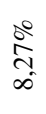 & $\begin{array}{l}\text { o̊ } \\
\text { فे } \\
\text { i }\end{array}$ & $\begin{array}{l}\stackrel{0}{ } \\
\text { ते } \\
\text { ñ }\end{array}$ \\
\hline & & $\begin{array}{l}\stackrel{2}{a} \\
\text { aे }\end{array}$ & $\begin{array}{l}\stackrel{0}{0} \\
\stackrel{0}{0} \\
\stackrel{i}{త}\end{array}$ & $\begin{array}{l}\stackrel{0}{0} \\
\infty \\
\infty \\
r\end{array}$ & $\stackrel{\stackrel{\circ}{\Im}}{\stackrel{\Im}{-}}$ & $\begin{array}{l}\stackrel{0}{~} \\
\text { à }\end{array}$ & $\begin{array}{l}\infty \\
\infty \\
\stackrel{\infty}{0}\end{array}$ & $\begin{array}{l}\stackrel{0}{0} \\
\text { हे } \\
\dot{ \pm}\end{array}$ & $\begin{array}{l}\stackrel{0}{n} \\
\stackrel{n}{n} \\
\stackrel{n}{n}\end{array}$ & $\begin{array}{l}\frac{\infty}{\infty} \\
\frac{\infty}{\infty}\end{array}$ & $\begin{array}{l}\text { ¿̊ } \\
\text { ర్ } \\
\text { i }\end{array}$ & $\begin{array}{l}\frac{0^{\circ}}{N} \\
\text { in }\end{array}$ \\
\hline & & $\begin{array}{l}\circ \\
2 \\
2\end{array}$ & $\begin{array}{l}\stackrel{2}{2} \\
\text { సे }\end{array}$ & $\begin{array}{l}\stackrel{0}{2} \\
\stackrel{\infty}{+} \\
\stackrel{+}{n}\end{array}$ & $\begin{array}{l}\stackrel{8}{I} \\
=\end{array}$ & $\stackrel{\stackrel{\circ}{N}}{\stackrel{i}{i}}$ & $\begin{array}{l}\frac{0}{\sigma} \\
\stackrel{2}{2}\end{array}$ & $\begin{array}{l}\frac{\partial}{2} \\
\text { ஸे } \\
\stackrel{n}{-}\end{array}$ & $\begin{array}{l}\stackrel{Ð}{\tilde{f}} \\
\stackrel{+}{ \pm}\end{array}$ & 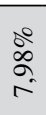 & 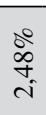 & $\begin{array}{c}\stackrel{0}{0} \\
\text { in } \\
\text { in }\end{array}$ \\
\hline & & $\begin{array}{l}\stackrel{2}{ } \\
\text { ă }\end{array}$ & $\begin{array}{l}\stackrel{0}{0} \\
\text { aे } \\
=\end{array}$ & $\frac{\stackrel{0}{N}}{\stackrel{N}{N}}$ & $\stackrel{8}{b}$ & $\begin{array}{l}0 \\
8 \\
0 \\
i \\
\text { i }\end{array}$ & $\frac{\stackrel{\circ}{\varrho}}{\stackrel{a}{2}}$ & $\begin{array}{l}\delta^{0} \\
\infty \\
\text { I }\end{array}$ & $\begin{array}{l}\stackrel{0}{ } \\
\infty \\
\stackrel{\infty}{n}\end{array}$ & $\begin{array}{l}\stackrel{0}{0} \\
\infty \\
\sim\end{array}$ & 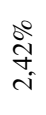 & $\begin{array}{l}\stackrel{0}{8} \\
8 \\
+\end{array}$ \\
\hline & \multirow{4}{*}{ 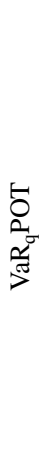 } & $\begin{array}{l}\stackrel{0}{2} \\
2 \\
\alpha\end{array}$ & $\begin{array}{l}\stackrel{0}{0} \\
\text { ஸ̂. } \\
\text { I }\end{array}$ & $\begin{array}{l}\frac{0}{\sigma} \\
\stackrel{2}{r}\end{array}$ & $\frac{\partial}{}$ & $\begin{array}{l}\stackrel{2}{2} \\
\text { ลे }\end{array}$ & $\begin{array}{l}0 \\
\infty \\
i n \\
n\end{array}$ & $\begin{array}{l}\stackrel{0}{8} \\
\text { + } \\
\dot{ \pm}\end{array}$ & 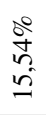 & $\begin{array}{l}\stackrel{0}{+} \\
\stackrel{+}{1} \\
\infty\end{array}$ & $\stackrel{\circ}{\stackrel{0}{i}}$ & $\begin{array}{l}\stackrel{2}{2} \\
\text { in }\end{array}$ \\
\hline & & $\begin{array}{l}\stackrel{2}{a} \\
\text { aे }\end{array}$ & $\begin{array}{l}80 \\
8 \\
\text { i }\end{array}$ & $\begin{array}{l}\stackrel{\circ}{\infty} \\
\stackrel{\infty}{r}\end{array}$ & $\begin{array}{l}\stackrel{0}{0} \\
\stackrel{n}{n}\end{array}$ & $\begin{array}{l}\stackrel{0}{ } \\
\text { ડे } \\
\text { i }\end{array}$ & $\begin{array}{l}\stackrel{8}{ \pm} \\
\stackrel{v}{2}\end{array}$ & $\begin{array}{l}\stackrel{\circ}{\circ} \\
\stackrel{-}{ \pm}\end{array}$ & $\begin{array}{l}\stackrel{0}{+} \\
\stackrel{+}{\sim} \\
\stackrel{2}{n}\end{array}$ & $\begin{array}{l}\stackrel{0}{0} \\
\stackrel{\text { }}{\infty}\end{array}$ & $\begin{array}{l}0 \\
80 \\
0 \\
i\end{array}$ & $\begin{array}{l}\stackrel{0}{2} \\
\text { in } \\
\text { n. }\end{array}$ \\
\hline & & $\begin{array}{l}\check{n}^{2} \\
2 \\
2\end{array}$ & $\begin{array}{l}\stackrel{\circ}{2} \\
\stackrel{=}{=}\end{array}$ & $\begin{array}{l}\stackrel{8}{0} \\
\stackrel{n}{r}\end{array}$ & $\begin{array}{l}\infty \\
\stackrel{\infty}{=}\end{array}$ & $\begin{array}{l}\stackrel{0}{?} \\
\text { i }\end{array}$ & 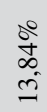 & $\begin{array}{l}\stackrel{0}{0} \\
\text { ปे } \\
\stackrel{2}{=}\end{array}$ & $\begin{array}{l}\stackrel{0}{0} \\
\text { m. } \\
\dot{ \pm}\end{array}$ & $\begin{array}{l}\stackrel{0}{0} \\
\dot{\infty}\end{array}$ & $\begin{array}{l}\stackrel{2}{+} \\
\stackrel{+}{i} \\
\text { i }\end{array}$ & $\begin{array}{l}\stackrel{0}{0} \\
\infty \\
\text { in }\end{array}$ \\
\hline & & $\begin{array}{l}\stackrel{\circ}{\sigma} \\
\text { ă }\end{array}$ & $\begin{array}{l}\overbrace{}^{\circ} \\
\stackrel{2}{0} \\
=\end{array}$ & $\begin{array}{l}\stackrel{\circ}{2} \\
\stackrel{2}{n}\end{array}$ & $\stackrel{8}{0}$ & $\begin{array}{l}\frac{0}{2} \\
\hat{n} \\
i\end{array}$ & $\frac{\stackrel{\partial}{ \pm}}{\stackrel{\sigma}{2}}$ & $\begin{array}{l}\stackrel{0}{2} \\
\stackrel{2}{a}\end{array}$ & $\begin{array}{l}\stackrel{0}{ } \\
\infty \\
\infty \\
\end{array}$ & $\begin{array}{l}\stackrel{\circ}{\Omega} \\
\text { న̆ }\end{array}$ & $\begin{array}{l}\stackrel{0}{0} \\
\infty \\
\text { î } \\
i\end{array}$ & \begin{tabular}{l}
$\stackrel{0}{+}$ \\
\multirow{+}{+}{}
\end{tabular} \\
\hline & \multicolumn{2}{|c|}{$\frac{1}{2}$} & $\begin{array}{l}\infty \\
\infty \\
\infty \\
\infty \\
\infty\end{array}$ & $\begin{array}{l}\stackrel{0}{2} \\
m \\
\text { nn }\end{array}$ & 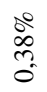 & $\begin{array}{l}\stackrel{0}{0} \\
\stackrel{0}{-}\end{array}$ & $\begin{array}{l}\stackrel{2}{2} \\
\stackrel{2}{r}\end{array}$ & 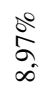 & $\begin{array}{l}\therefore 0 \\
\hat{n} \\
a\end{array}$ & $\begin{array}{l}80 \\
8 \\
8\end{array}$ & $\stackrel{\stackrel{\circ}{N}}{\approx}$ & $\begin{array}{l}\infty \\
\infty \\
\infty \\
i\end{array}$ \\
\hline & \multicolumn{2}{|c|}{ 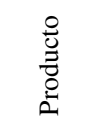 } & $\ll$ & $\infty$ & $u$ & $\theta$ & 피 & I工 & ט & $I$ & - & $\sim$ \\
\hline
\end{tabular}


inapreciables con las estimaciones históricas de los parámetros de riesgos. Los efectos benéficos de esta mayor capacidad se traducen en dos ventajas sobre las anteriores dos cópulas: la posibilidad de materializar las mejores estimaciones de $P E$ y $V a R_{q}$ en una medida de agregación total y la oportunidad de medir los beneficios derivados de la diversificación del portafolio.

En lo que corresponde a la primera ventaja, hay poco que agregar: la Tabla 10 proporciona estimaciones de riesgo para el portafolio total que no son provistas por las Tablas 8 y $9 .{ }^{12}$ Y esta es una diferencia muy importante. La otra ventaja se descubre al analizar los últimos dos renglones de la Tabla 10, donde se incluye, respectivamente, la suma aritmética del $V_{a} R_{q}$ marginal de todos los productos ponderada por el saldo total (Total P1) y la raíz de la suma de los cuadrados de $V a R_{q}$ (Total P0), es decir, bajo el supuesto de correlación cero entre productos. En efecto, al comparar las estimaciones de $V a R_{q}$ del portafolio total al 99,5\% con la suma aritmética de $V a R_{q}$ 's individuales se obtiene una diferencia promedio de alrededor del $0,4 \%$, que es precisamente el beneficio por diversificación.

Ahora bien, hasta aquí las comparaciones entre las estimaciones del $V a R_{q}$ se han llevado a cabo sólo a escala puntual y tomando como referencia el benchkmark histórico de la Tabla 3. Sin embargo, para dar cuenta de los efectos más generales que tienen las familias de cópulas ajustadas sobre los métodos de cálculo de $V_{a} R_{q}$ es conveniente comparar también los intervalos de confianza de tales estimadores. En la práctica, los intervalos de confianza para las estimaciones del valor en riesgo se construyen suponiendo, entre otras cosas, normalidad y algunas propiedades asintóticas de la distribución de la varianza muestral (ver Dowd, 2000). En nuestro caso, la forma asimétrica de las distribuciones de pérdidas y el hecho de que los estimadores de los parámetros de las distribuciones calculados con POT y Beta son obtenidos por máxima verosimilitud nos obliga a obviar estas prácticas convencionales y a caracterizar la distribución límite de los estimadores de $V a R_{q}$ con métodos alternativos, tales como el "delta" o el de cociente de verosimilitud generalizado. En concreto, decidimos adoptar este último método porque permite obtener, primero, las llamadas curvas de log-verosimilitud fiducial y, luego, los intervalos de confianza, en general asimétricos, sin tener que lidiar con las deficiencias propias de la aplicación del método delta (ver Apéndice 2). ${ }^{13}$

La Tabla 11 contiene los extremos inferiores y superiores de los intervalos de confianza de las distribuciones de pérdida generadas por las cópulas multivariadas y que son usados para estimar los valores de $\mathrm{VaR}_{99,5 \%}$ por los métodos POT y Beta.

12 Las estimaciones de $P E$ y VaRq por producto obtenidas con la cópula $t$ de Student agrupada son muy parecidas a las desplegadas por las dos tablas anteriores debido a que se emplea una cópula t simétrica para cada producto en los tres ajustes. La diferencia es que, por construcción, la cópula t agrupada ofrece una medida consolidada del riesgo que no es posible derivar de las dos anteriores cópulas, que fueron ajustadas por producto.

13 Entre las ventajas de usar este método destaca el hecho de garantizar que los intervalos resultantes se encuentren contenidos en la distribución subyacente, además de incluir en la estadística de prueba tanto la incertidumbre de $\hat{\xi}$ y $\hat{\beta}$ como de $\mathrm{N}_{\mathrm{u}} / \mathrm{n}$, la cual sirve como estimador de la cola de la distribución en el método POT. 


\begin{tabular}{|c|c|c|c|c|c|c|c|c|}
\hline \multirow{4}{*}{ 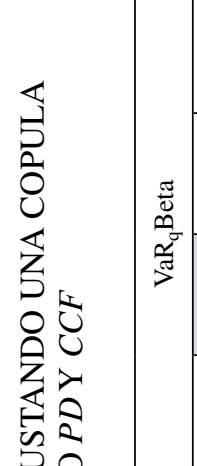 } & \multirow{4}{*}{ 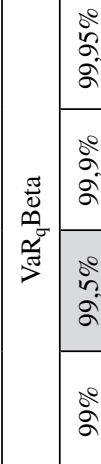 } & 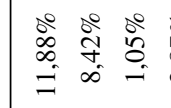 & 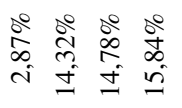 & $\begin{array}{ll}0 & 8 \\
\vec{N} & 0 \\
\infty & 1\end{array}$ & $\begin{array}{l}\stackrel{0}{8} \\
\text { in } \\
i\end{array}$ & & $\begin{array}{l}\stackrel{0}{\infty} \\
\text { in }\end{array}$ & 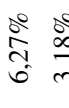 \\
\hline & & 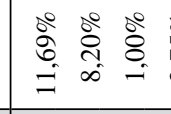 & 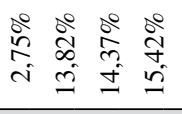 & $\begin{array}{ll}0 & 0 \\
0 & 0 \\
0 & 1\end{array}$ & $\begin{array}{l}\stackrel{0}{0} \\
\text { ñ } \\
i\end{array}$ & $\begin{array}{l}0 \\
\vdots \\
\infty \\
+ \\
+\end{array}$ & $\begin{array}{l}\text { O9 } \\
\text { di } \\
\text { in }\end{array}$ & $\begin{array}{ll}0 & 0 \\
0 & 8 \\
6 & 9\end{array}$ \\
\hline & & 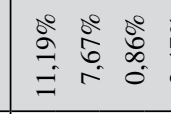 & 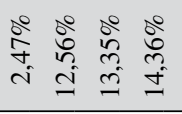 & $\begin{array}{ll}\therefore 0 & 8 \\
0 & 0 \\
0 & 0\end{array}$ & $\begin{array}{l}\stackrel{0}{0} \\
\text { a } \\
\text { i }\end{array}$ & \begin{tabular}{l}
$\stackrel{0}{\infty}$ \\
$\stackrel{+}{+}$ \\
\multirow{+}{*}{}
\end{tabular} & $\frac{8}{m}$ & $\begin{array}{ll}0 & 0 \\
i & \infty \\
\text { in } & \infty\end{array}$ \\
\hline & & 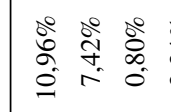 & 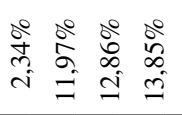 & $\begin{array}{l}\stackrel{0}{2} \\
\sim \\
\sim\end{array}$ & $\begin{array}{l}\text { o̊ } \\
\text { ç } \\
\text { in }\end{array}$ & $\begin{array}{l}\stackrel{0}{0} \\
\stackrel{m}{+}\end{array}$ & $\frac{i}{i n}$ & 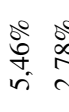 \\
\hline 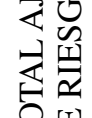 & $\begin{array}{l}\stackrel{\circ}{2} \\
2\end{array}$ & 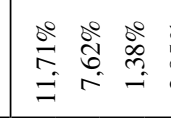 & 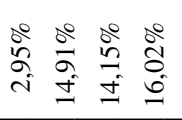 & $\begin{array}{ll}0 & 0 \\
\pi & 1 \\
\infty & 1\end{array}$ & $\begin{array}{l}\stackrel{0}{2} \\
i n \\
i\end{array}$ & $\begin{array}{l}\stackrel{0}{2} \\
\text { ì }\end{array}$ & $\begin{array}{l}\vdots \\
\vdots\end{array}$ & 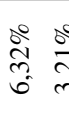 \\
\hline 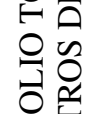 & $\begin{array}{l}\text { aे } \\
\text { aे }\end{array}$ & 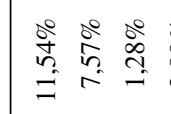 & 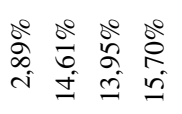 & $\frac{8}{0}$ & $\begin{array}{l}\stackrel{0}{0} \\
\text { in } \\
i\end{array}$ & $\begin{array}{l}\stackrel{2}{ \pm} \\
\text { in } \\
\text { in }\end{array}$ & $\begin{array}{l}\dot{\infty} \\
\dot{\infty} \\
\dot{n}\end{array}$ & $\begin{array}{ll}\stackrel{0}{0} & \text { in } \\
\text { त̂ } & \text { in } \\
0 & \text { ल }\end{array}$ \\
\hline 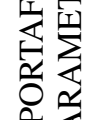 & $\begin{array}{l}\overbrace{}^{2} \\
2\end{array}$ & $\begin{array}{l}\stackrel{0}{0} \stackrel{0}{0} \\
\stackrel{2}{=} \\
= \\
=\end{array}$ & 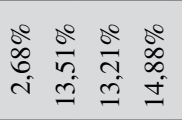 & $\begin{array}{ll}0 & 8 \\
2 & 1 \\
2 & 0\end{array}$ & $\begin{array}{l}\stackrel{0}{a} \\
\text { ma } \\
i\end{array}$ & $\begin{array}{l}\stackrel{0}{0} \\
2 \\
\sigma\end{array}$ & $\begin{array}{l}\dot{0} \\
i n \\
i\end{array}$ & $\begin{array}{ll}0 & 5 \\
\infty & 8 \\
\infty & 0\end{array}$ \\
\hline 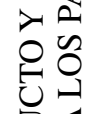 & $\begin{array}{l}\stackrel{2}{\circ} \\
\text { ă }\end{array}$ & 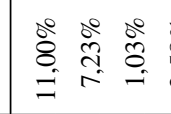 & 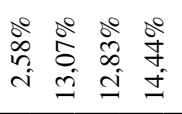 & $\begin{array}{ll}\stackrel{0}{1} & 5 \\
\infty & 5 \\
& 0\end{array}$ & $\frac{o}{m}$ & $\begin{array}{l}\stackrel{0}{2} \\
\infty \\
\infty \\
+\end{array}$ & $\begin{array}{l}\stackrel{0}{0} \\
m \\
i\end{array}$ & 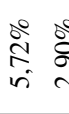 \\
\hline 官㝴 & $\begin{array}{l}\stackrel{\circ}{2} \\
\check{\sigma}\end{array}$ & 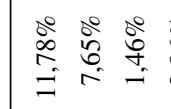 & 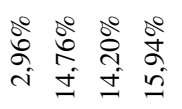 & $\frac{\vdots}{9}$ & $\begin{array}{l}\stackrel{0}{8} \\
i n \\
i\end{array}$ & $\begin{array}{l}\frac{0}{N} \\
\text { in }\end{array}$ & $\begin{array}{l}\circ 0 \\
\infty \\
\text { in }\end{array}$ & 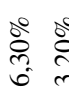 \\
\hline 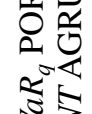 & $\begin{array}{l}\text { aे } \\
\text { aे }\end{array}$ & 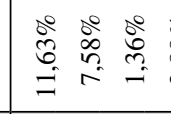 & 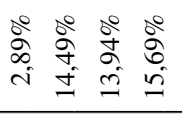 & $\begin{array}{l}0 \\
0 \\
\infty \\
\infty\end{array}$ & $\begin{array}{l}\stackrel{0}{0} \\
\text { on } \\
i\end{array}$ & $\begin{array}{l}\stackrel{0}{0} \\
\text { ñ }\end{array}$ & $\begin{array}{l}8 \\
\infty \\
\text { in }\end{array}$ & 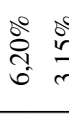 \\
\hline 式 & $\begin{array}{l}20 \\
2 \\
2\end{array}$ & $\begin{array}{l}\stackrel{\circ}{\circ} \stackrel{\circ}{\check{2}} \\
\stackrel{2}{\leftrightarrows} \\
=\end{array}$ & 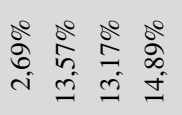 & $\begin{array}{ll}0 & 0 \\
8 & 0 \\
0 & 1 \\
\infty & 0\end{array}$ & $\begin{array}{l}\stackrel{0}{m} \\
\text { in } \\
i\end{array}$ & $\begin{array}{l}\frac{0}{0} \\
0 \\
i\end{array}$ & $\begin{array}{l}\stackrel{0}{N} \\
\text { ñ } \\
\text { nn }\end{array}$ & $\begin{array}{ll}\circ & 0 \\
8 & 8 \\
\text { in } & 8\end{array}$ \\
\hline 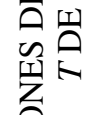 & ڤ̊ & 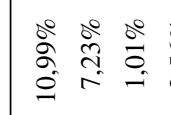 & 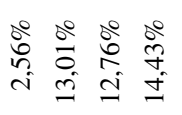 & $\begin{array}{ll}\infty & 0 \\
\infty & 0 \\
\infty & 1\end{array}$ & $\begin{array}{l}\stackrel{0}{0} \\
\text { co } \\
i\end{array}$ & $\begin{array}{l}\dot{0} \\
\dot{0} \\
\dot{+}\end{array}$ & $\begin{array}{l}80 \\
\text { in } \\
\text { in }\end{array}$ & $\begin{array}{ll}0 & 0 \\
\pi & 0 \\
\text { in } & 0\end{array}$ \\
\hline$\sum_{\Sigma}^{U}$ & ț & 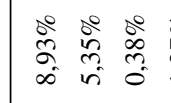 & 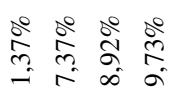 & $\begin{array}{lll}0 & 0 \\
0 & 0 \\
0 & 0\end{array}$ & $\stackrel{\stackrel{0}{\stackrel{2}{=}}}{=}$ & $\begin{array}{l}\stackrel{0}{2} \\
\text { के } \\
\text { i }\end{array}$ & $\begin{array}{l}0 \\
0 \\
\infty \\
\text { mi }\end{array}$ & $\begin{array}{l}0^{0} \\
\infty \\
\infty \\
m\end{array}$ \\
\hline کి & $\begin{array}{l}0 \\
\frac{0}{0} \\
\frac{7}{0} \\
0\end{array}$ & $\varangle \oplus U$ & 口 I I U & 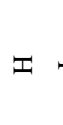 & - & $\neg$ & స్తేర & $\begin{array}{ll}\overline{2} & 0 \\
\bar{\pi} & \frac{\pi}{0} \\
\frac{\pi}{0} & \frac{\pi}{6}\end{array}$ \\
\hline
\end{tabular}




\section{FIGURA 3}

HISTOGRAMAS DE LAS PERDIDAS SIMULADAS POR PRODUCTO Y PORTAFOLIO TOTAL AJUSTANDO UNA COPULA $T$ DE STUDENT AGRUPADA A LOS PARAMETROS PDY $C C F$
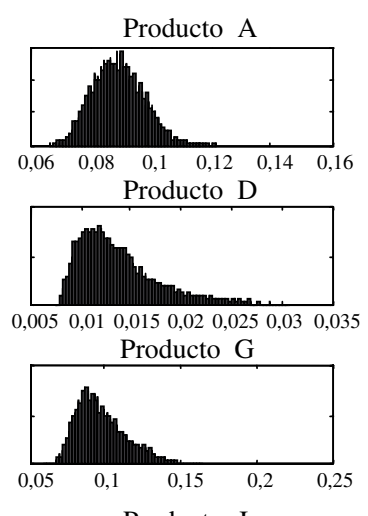

Producto J

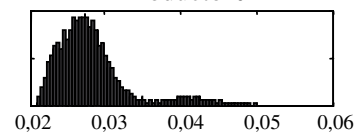

Producto B
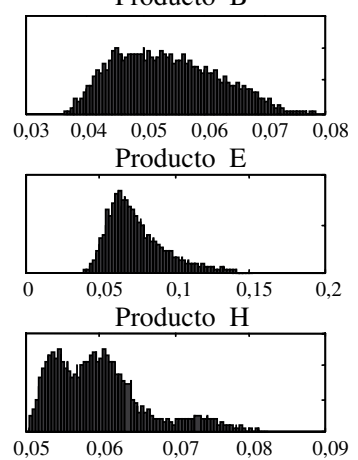

Portafolio Total
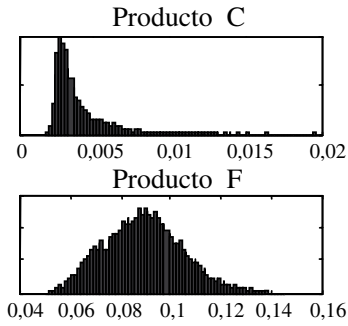

Producto I
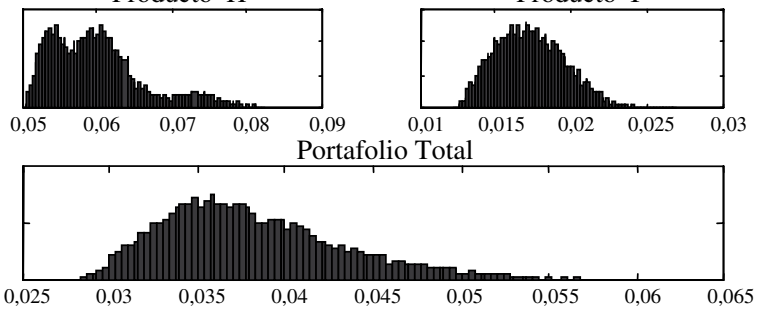

En general, la tabla muestra que mientras la estructura de dependencia dada por la cópula t agrupada presenta los intervalos de confianza con mayor amplitud, la cópula t simétrica exhibe los más cortos. Del mismo modo, se puede señalar que aun cuando no hay diferencia entre las áreas de la cola capturadas por ambas cópulas, la t agrupada tiene el efecto de aumentar ligeramente la variabilidad de la estimación de los parámetros en los altos cuantiles y, de esa manera, capturar el efecto de escenarios más extremos. Finalmente, cabe notar que si bien los intervalos de confianza son asimétricos respecto de sus estimaciones puntuales en las Tablas 8, 9 y 10, el método POT incorpora una variabilidad superior sobre la medida de riesgo.

\subsection{Pruebas de bondad de ajuste}

Para establecer estadísticamente la bondad de ajuste de cada cópula se emplea el estadístico $Q$ de formas bilineales propuesto por Panchenko (2005). Este estadístico es una medida de discrepancia entre la cópula empírica y alguna otra familia de cópulas empleada, en la que valores pequeños indican un mejor ajuste de estas últimas. En la Tabla 12 se presentan los valores promedio de las distancias $Q$ por producto y portafolio total. Un bosquejo general sobre el cálculo de dicha medida de discrepancia entre distribuciones multivariadas aparece en el Apéndice A4.

Los valores de la estadística de prueba $Q$ revelan que mientras la cópula $t$ de Student agrupada ajusta muy bien los datos, las cópulas de independencia y gaussiana ofrecen, 


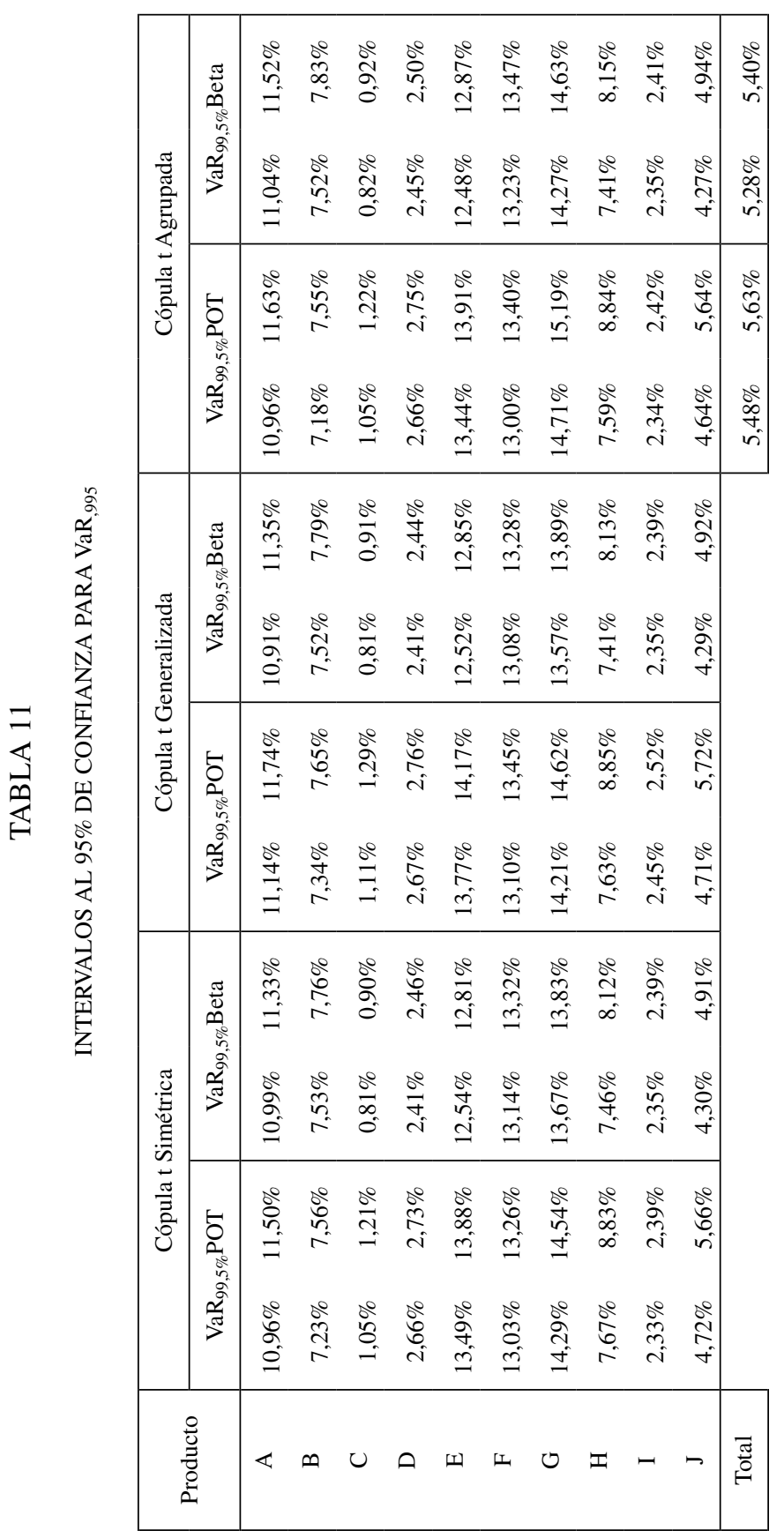




\section{TABLA 12}

DISTANCIAS $Q$ (ESTADISTICA DE PRUEBA) PARA CADA TIPO DE COPULAS AJUSTADO

\begin{tabular}{|c|c|c|c|c|c|c|}
\hline Producto & $\begin{array}{c}\text { Cópula } \\
\text { Independencia } \\
\text { portafolio total }\end{array}$ & $\begin{array}{c}\text { Cópula } \\
\text { Gaussiana } \\
\text { portafolio } \\
\text { total }\end{array}$ & $\begin{array}{c}\text { Cópula t } \\
\text { Simétrica } \\
\text { portafolio } \\
\text { total }\end{array}$ & $\begin{array}{c}\text { Cópula t } \\
\text { Simétrica } \\
\text { por producto }\end{array}$ & $\begin{array}{c}\text { Cópula t } \\
\text { Generalizada } \\
\text { por producto }\end{array}$ & $\begin{array}{c}\text { Cópula t } \\
\text { Agrupada }\end{array}$ \\
\hline A & 0,10415 & 0,07264 & 0,04061 & 0,04016 & 0,04069 & 0,03980 \\
B & 0,09646 & 0,06839 & 0,03817 & 0,03786 & 0,03793 & 0,03670 \\
C & 0,09191 & 0,06615 & 0,03667 & 0,03662 & 0,03669 & 0,03600 \\
D & 0,09699 & 0,06858 & 0,03841 & 0,03791 & 0,03807 & 0,03700 \\
E & 0,10004 & 0,07006 & 0,03916 & 0,03866 & 0,03886 & 0,03810 \\
F & 0,09482 & 0,06797 & 0,03774 & 0,03752 & 0,03754 & 0,03670 \\
G & 0,09040 & 0,06519 & 0,03615 & 0,03612 & 0,03612 & 0,03580 \\
H & 0,10676 & 0,07413 & 0,04152 & 0,04152 & 0,04134 & 0,03970 \\
I & 0,11059 & 0,07632 & 0,04255 & 0,04275 & 0,04463 & 0,04080 \\
J & 0,10658 & 0,07577 & 0,04206 & 0,04213 & 0,04223 & 0,04110 \\
\hline Total & 0,08873 & 0,06400 & 0,03549 & 0,03549 & 0,03549 & 0,03460 \\
\hline
\end{tabular}

en cambio, las simulaciones más pobres de todas las familias de cópulas utilizadas. Las diferencias son notorias pues los valores de $Q$ para estas dos últimas cópulas resultan ser de más del doble que los registrados para la t agrupada: un resultado que da pie a pensar que si se modela la estructura de dependencia de $P D$ y $C C F$ con la cópula $t$ de Student agrupada y se elimina el supuesto de constancia de $L G D$, es posible obtener mejores estimaciones de $E S_{q}$ y $V a R_{q}$ que con los ajustes anteriores.

Los resultados de las Tablas 13 y 14 parecen confirmar tal presunción, en particular cuando se modela a $L G D$ con la cópula simétrica $t$ de Student. En ese caso las estimaciones obtenidas por el método POT resultan más consistentes y confiables para niveles de $q$ cercanos a 1 que aquellas registradas por los valores históricos de la Tabla $3 .{ }^{14}$

La explicación a esta mejora reside en las propiedades asintóticas de los estimadores de $\operatorname{VaR}_{q}$ y $E S_{q}$ obtenidos por el método POT para niveles de confianza ligeramente inferiores al 100\% (Embrechts et al., 1997; y Díaz, 2003). Esto se puede comprobar al comparar entre sí las estimaciones de las medidas de riesgo $V_{a} R_{q}$ y $E S_{q}$ de la distribución de pérdidas del portafolio total obtenidas con los métodos POT, Empírico y Beta. Las estimaciones basadas en el método POT (en gris claro) muestran consistencia con las estimaciones empíricas (en gris oscuro) cuando se utilizan niveles de confianza inferiores al 0,999 y estabilidad frente al estimador empírico para niveles de confianza superiores. Y es que una vez fijado el número de simulaciones, el estimador sólo es consistente para niveles de confianza $q$ inferiores a los que se observan

14 Con el fin de estresar las distribuciones marginales del modelo, las densidades kernel se ajustan con el doble del ancho de banda empleado en los ejercicios anteriores. 


\begin{tabular}{|c|c|c|c|c|c|c|c|c|c|c|c|c|c|c|c|}
\hline \multirow{14}{*}{ 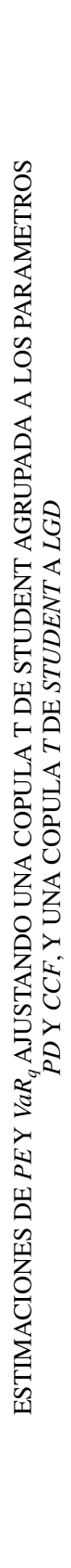 } & \multirow{4}{*}{ 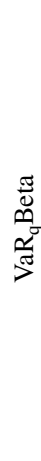 } & $\begin{array}{l}\stackrel{2}{\alpha} \\
\stackrel{\alpha}{\alpha} \\
\text { a }\end{array}$ & 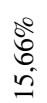 & $\begin{array}{l}\stackrel{0}{n} \\
\stackrel{n}{=} \\
=\end{array}$ & $\begin{array}{l}\frac{0}{\pi} \\
\text { î }\end{array}$ & $\begin{array}{l}\stackrel{0}{0} \\
\text { ले }\end{array}$ & 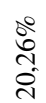 & $\begin{array}{l}\delta_{0}^{0} \\
\stackrel{0}{0} \\
\text { a }\end{array}$ & $\begin{array}{l}\stackrel{0}{2} \\
\stackrel{m}{=} \\
=\end{array}$ & $\begin{array}{l}0 \\
\stackrel{0}{0} \\
\pm \\
\pm\end{array}$ & 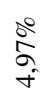 & $\begin{array}{l}\stackrel{2}{2} \\
\stackrel{2}{a}\end{array}$ & 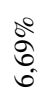 & $\begin{array}{l}\delta_{0}^{0} \\
\stackrel{0}{0} \\
\infty\end{array}$ & $\begin{array}{l}\stackrel{\circ}{\sigma} \\
\text { ले }\end{array}$ \\
\hline & & $\begin{array}{l}\stackrel{\circ}{\alpha} \\
\text { aे }\end{array}$ & $\begin{array}{l}\stackrel{\circ}{a} \\
\stackrel{n}{n}\end{array}$ & $\begin{array}{l}\stackrel{0}{0} \\
\stackrel{0}{=}\end{array}$ & $\begin{array}{l}\text { o̊ } \\
\text { ô } \\
\text { i }\end{array}$ & $\begin{array}{l}\stackrel{0}{\circ} \\
\text { m }\end{array}$ & $\begin{array}{l}\stackrel{0}{\infty} \\
\stackrel{0}{2} \\
\stackrel{2}{2}\end{array}$ & $\begin{array}{l}\stackrel{0}{0} \\
\text { กิ } \\
\infty\end{array}$ & $\begin{array}{l}0 \\
0 \\
\infty \\
0 \\
0\end{array}$ & $\begin{array}{l}\stackrel{0}{8} \\
8 \\
\pm\end{array}$ & $\frac{\stackrel{\partial}{\sigma}}{\underset{\nabla}{*}}$ & $\begin{array}{l}\stackrel{0}{0} \\
\infty \\
\infty\end{array}$ & $\begin{array}{l}\stackrel{0}{0} \\
\text { aे } \\
\text { ó }\end{array}$ & $\begin{array}{l}\stackrel{0}{0} \\
\text { ल. } \\
\infty\end{array}$ & $\begin{array}{l}b^{0} \\
\infty \\
\infty \\
\text { r. }\end{array}$ \\
\hline & & $\begin{array}{l}\text { i̊ } \\
\text { ลे }\end{array}$ & $\begin{array}{l}\delta^{\circ} \\
0 \\
\dot{ \pm}\end{array}$ & $\begin{array}{l}\frac{2}{5} \\
\text { aे }\end{array}$ & 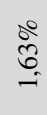 & $\begin{array}{l}\stackrel{0}{0} \\
\text { ஸे } \\
\text { ñ }\end{array}$ & $\begin{array}{l}\theta_{0}^{0} \\
\infty \\
0 \\
0\end{array}$ & $\begin{array}{l}\stackrel{0}{\infty} \\
\stackrel{+}{+} \\
\stackrel{0}{0}\end{array}$ & $\begin{array}{l}\stackrel{0}{\infty} \\
\stackrel{+}{+} \\
\stackrel{+}{2}\end{array}$ & 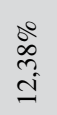 & $\begin{array}{l}\stackrel{0}{0} \\
\infty \\
\stackrel{+}{+} \\
\end{array}$ & $\frac{0^{\circ}}{+2}$ & $\begin{array}{l}\delta^{\circ} \\
\text { nू }\end{array}$ & 字 & 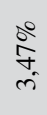 \\
\hline & & ڤ̊ & $\begin{array}{l}\dot{8} \\
\dot{0} \\
\dot{0} \\
\text { m. }\end{array}$ & $\begin{array}{l}\stackrel{0}{~} \\
\stackrel{n}{\sigma} \\
\sigma\end{array}$ & 字 & $\begin{array}{l}\stackrel{0}{0} \\
\stackrel{2}{\alpha} \\
\text { i }\end{array}$ & $\begin{array}{l}\text { 号 } \\
\text { in } \\
\text { in }\end{array}$ & $\begin{array}{l}\delta^{\circ} \\
\text { రై } \\
\text { ñ }\end{array}$ & $\begin{array}{l}00 \\
\dot{0} \\
\dot{ \pm}\end{array}$ & $\begin{array}{l}\stackrel{0}{0} \\
\tilde{6} \\
=\end{array}$ & $\frac{\stackrel{\circ}{g}}{\stackrel{m}{m}}$ & $\begin{array}{l}80 \\
\text { ᄋ̊ } \\
0\end{array}$ & $\frac{80}{i 0}$ & \begin{tabular}{l}
$\stackrel{0}{0}$ \\
\multirow{0}{0}{}
\end{tabular} & $\begin{array}{l}\stackrel{\circ}{\circ} \\
\text { ते } \\
\text { ले }\end{array}$ \\
\hline & \multirow{4}{*}{ 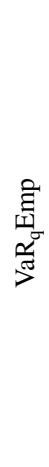 } & $\begin{array}{l}\stackrel{2}{2} \\
\stackrel{\alpha}{\alpha} \\
\text { a }\end{array}$ & $\begin{array}{l}80 \\
\stackrel{8}{2} \\
\text { I }\end{array}$ & \begin{tabular}{l}
$\stackrel{0}{0}$ \\
\multirow{0}{*}{} \\
$=$
\end{tabular} & $\begin{array}{l}\stackrel{0}{0} \\
\dot{\infty} \\
i \\
i\end{array}$ & 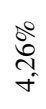 & $\begin{array}{l}\stackrel{ }{\stackrel{2}{*}} \\
\text { a }\end{array}$ & $\underset{\infty}{\stackrel{0}{\infty}}$ & $\begin{array}{l}00 \\
\stackrel{0}{2} \\
\stackrel{2}{=}\end{array}$ & $\begin{array}{l}\stackrel{0}{0} \\
\text { + } \\
\pm\end{array}$ & $\begin{array}{l}\stackrel{8}{0} \\
\underset{f}{*}\end{array}$ & $\begin{array}{l}\stackrel{0}{0} \\
\cong \\
=\end{array}$ & 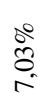 & $\frac{\stackrel{8}{2}}{\infty}$ & $\begin{array}{l}b^{0} \\
0 \\
\dot{f}\end{array}$ \\
\hline & & $\begin{array}{l}\text { ळ̊ } \\
\text { aे }\end{array}$ & $\begin{array}{l}\stackrel{\circ}{\circ} \\
\stackrel{0}{6}\end{array}$ & 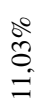 & $\begin{array}{l}\stackrel{2}{2} \\
\text { i }\end{array}$ & $\begin{array}{l}8_{0}^{0} \\
\underset{f}{f}\end{array}$ & $\begin{array}{l}\stackrel{\circ}{\Xi} \\
\vec{d}\end{array}$ & \begin{tabular}{l}
$\stackrel{8}{+}$ \\
\multirow{2}{0}{} \\
$\infty$
\end{tabular} & $\begin{array}{l}\stackrel{0}{\infty} \\
\text { Ñ } \\
\text { I }\end{array}$ & $\begin{array}{l}\stackrel{0}{0} \\
\infty \\
0 \\
0\end{array}$ & $\begin{array}{l}0^{0} \\
\text { d } \\
\dot{f}\end{array}$ & $\begin{array}{l}00 \\
\stackrel{n}{n} \\
0 \\
0\end{array}$ & $\stackrel{\stackrel{\circ}{N}}{\stackrel{0}{0}}$ & $\begin{array}{l}\stackrel{\infty}{\circ} \\
\stackrel{+}{\infty} \\
\infty\end{array}$ & $\frac{\partial}{\sigma}$ \\
\hline & & $\begin{array}{l}\text { के } \\
\text { ลे }\end{array}$ & $\begin{array}{l}\dot{0}^{\circ} \\
\infty \\
\dot{ \pm}\end{array}$ & $\frac{\stackrel{0}{\Sigma}}{0}$ & $\begin{array}{l}\stackrel{0}{2} \\
\stackrel{n}{i} \\
i\end{array}$ & $\begin{array}{l}\frac{0}{n} \\
\text { n }\end{array}$ & $\begin{array}{l}\text { bo } \\
\text { a } \\
\text { a }\end{array}$ & 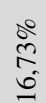 & $\frac{\stackrel{0}{\stackrel{0}{0}}}{\stackrel{0}{0}}$ & $\begin{array}{l}\frac{0}{2} \\
\text { ก } \\
\text { In }\end{array}$ & 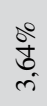 & $\frac{0^{0}}{n}$ & $\frac{\check{\sigma}^{\circ}}{\sigma_{0}^{\circ}}$ & $\begin{array}{l}\stackrel{0}{6} \\
\stackrel{6}{r}\end{array}$ & $\begin{array}{l}\frac{0}{0} \\
i n \\
m\end{array}$ \\
\hline & & $\begin{array}{l}\text { ลे } \\
\text { aे }\end{array}$ & $\begin{array}{l}\stackrel{\circ}{\sigma} \\
\text { ळे }\end{array}$ & $\begin{array}{l}\partial^{0} \\
\infty \\
0 \\
\sigma\end{array}$ & $\begin{array}{l}\stackrel{0}{0} \\
\text { aे } \\
\text { in }\end{array}$ & $\begin{array}{l}\stackrel{2}{2} \\
\stackrel{n}{m}\end{array}$ & \begin{tabular}{l}
$\stackrel{0}{f}$ \\
\multirow{f}{*}{}
\end{tabular} & $\begin{array}{l}\frac{0}{0} \\
\infty \\
n \\
n\end{array}$ & 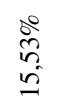 & $\begin{array}{l}\stackrel{0}{*} \\
=\end{array}$ & $\begin{array}{l}\stackrel{0}{0} \\
\stackrel{n}{n} \\
\text { n. }\end{array}$ & 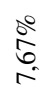 & $\begin{array}{l}\stackrel{0}{2} \\
2 \\
\text { nn }\end{array}$ & $\begin{array}{l}\stackrel{\Delta}{N} \\
\stackrel{N}{r}\end{array}$ & $\frac{\delta^{\circ}}{\sigma_{0}}$ \\
\hline & \multirow{4}{*}{$\begin{array}{l}5 \\
0 \\
0 \\
\frac{1}{\pi} \\
>\end{array}$} & $\begin{array}{l}\stackrel{\circ}{2} \\
\stackrel{\alpha}{\alpha} \\
\alpha\end{array}$ & $\begin{array}{l}\stackrel{\Delta}{2} \\
\stackrel{2}{=}\end{array}$ & $\stackrel{\stackrel{\circ}{2}}{\stackrel{2}{=}}$ & i̊ & $\stackrel{\stackrel{0}{\sim}}{\stackrel{\sim}{\sim}}$ & $\begin{array}{l}\text { ठ̊ } \\
\text { ᄋे } \\
\text { ते }\end{array}$ & $\begin{array}{l}\frac{0}{0} \\
n \\
\infty \\
\infty\end{array}$ & 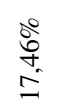 & $\begin{array}{l}\stackrel{\circ}{1} \\
\stackrel{ \pm}{ \pm}\end{array}$ & $\begin{array}{l}\stackrel{\circ}{0} \\
\stackrel{+}{+}\end{array}$ & $\begin{array}{l}\stackrel{0}{0} \\
\text { ఠ్ } \\
=\end{array}$ & $\begin{array}{l}\text { ळ̊ } \\
\text { â }\end{array}$ & $\begin{array}{l}\stackrel{0}{0} \\
\stackrel{0}{0} \\
\infty\end{array}$ & $\begin{array}{l}\stackrel{\circ}{\sigma} \\
\text { ले }\end{array}$ \\
\hline & & $\begin{array}{l}\stackrel{\circ}{\alpha} \\
\text { aे }\end{array}$ & $\begin{array}{l}\stackrel{0}{0} \\
\stackrel{n}{\sigma} \\
0\end{array}$ & $\begin{array}{l}\stackrel{\circ}{0} \\
\text { ડ } \\
=\end{array}$ & $\begin{array}{l}\stackrel{0}{0} \\
\infty \\
\infty \\
i\end{array}$ & $\underset{\stackrel{0}{\circ}}{\stackrel{8}{\circ}}$ & $\begin{array}{l}\stackrel{0}{\circ} \\
\dot{0} \\
\text { ते }\end{array}$ & $\begin{array}{l}\stackrel{0}{0} \\
\dot{0} \\
\infty\end{array}$ & $\begin{array}{l}\stackrel{0}{\cong} \\
\stackrel{-}{=}\end{array}$ & $\begin{array}{l}00 \\
\infty \\
0 \\
\stackrel{0}{0}\end{array}$ & $\begin{array}{l}\stackrel{2}{2} \\
\text { ले }\end{array}$ & $\begin{array}{l}\stackrel{0}{2} \\
\stackrel{2}{0} \\
\stackrel{0}{0}\end{array}$ & $\frac{8}{\frac{8}{7}}$ & $\begin{array}{l}\stackrel{0}{2} \\
\text { ले } \\
\infty\end{array}$ & $\begin{array}{l}\stackrel{0}{0} \\
\infty \\
\infty \\
\infty\end{array}$ \\
\hline & & $\begin{array}{l}i^{2} \\
\text { aे }\end{array}$ & $\begin{array}{l}\stackrel{\circ}{2} \\
\dot{ \pm}\end{array}$ & $\begin{array}{l}\stackrel{0}{0} \\
\text { กิ } \\
0\end{array}$ & $\begin{array}{l}\stackrel{2}{\check{~}} \\
\text { ते }\end{array}$ & $\begin{array}{l}\stackrel{0}{ } \\
\text { ñ } \\
m\end{array}$ & $\begin{array}{l}\text { ठ̊ } \\
8 \\
\text { ᄋ. }\end{array}$ & $\begin{array}{l}00 \\
0 \\
0 \\
0\end{array}$ & $\begin{array}{l}00 \\
0 \\
0 \\
0\end{array}$ & $\begin{array}{l}0 \\
0 \\
\text { I } \\
\text { di }\end{array}$ & $\begin{array}{l}\text { ठ̊̊ } \\
\text { రి } \\
\text { m. }\end{array}$ & $\begin{array}{l}\stackrel{0}{0} \\
\infty \\
\infty \\
\infty\end{array}$ & $\frac{\sigma^{0}}{\sqrt{0}}$ & 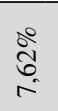 & $\begin{array}{l}\frac{8}{n} \\
\text { n }\end{array}$ \\
\hline & & $\frac{8}{\sigma}$ & 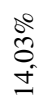 & $\frac{\stackrel{a}{~}}{\stackrel{\sigma}{a}}$ & $\begin{array}{l}80 \\
8 \\
0 \\
i\end{array}$ & $\begin{array}{l}\stackrel{0}{n} \\
\stackrel{n}{n} \\
\stackrel{n}{n}\end{array}$ & $\begin{array}{l}\stackrel{0}{0} \\
\stackrel{n}{n} \\
\cong\end{array}$ & $\begin{array}{l}\stackrel{0}{0} \\
\stackrel{-}{1} \\
\text { in }\end{array}$ & $\begin{array}{l}\frac{0}{n} \\
n \\
n\end{array}$ & $\begin{array}{l}\stackrel{0}{0} \\
\infty \\
= \\
=\end{array}$ & $\begin{array}{l}\stackrel{\circ}{a} \\
\stackrel{+}{+} \\
\text { m. }\end{array}$ & $\stackrel{\circ}{\stackrel{2}{2}}$ & $\begin{array}{l}\frac{b}{2} \\
\text { nू } \\
n\end{array}$ & $\stackrel{\stackrel{\Delta}{N}}{\stackrel{i}{r}}$ & 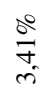 \\
\hline & \multicolumn{2}{|c|}{$\underline{\underline{2}}$} & $\frac{\partial 0}{\partial}$ & \begin{tabular}{l}
$\stackrel{0}{\infty}$ \\
\multirow{2}{*}{} \\
$i$
\end{tabular} & $\stackrel{\stackrel{0}{m}}{\stackrel{m}{0}}$ & $\stackrel{\circ 0}{\Rightarrow}$ & $\frac{20}{6}$ & $\begin{array}{l}\stackrel{0}{0} \\
\text { ô } \\
\text { a }\end{array}$ & $\begin{array}{l}\stackrel{0}{0} \\
\infty \\
a\end{array}$ & $\begin{array}{l}\stackrel{0}{0} \\
\text { oे } \\
0\end{array}$ & $\stackrel{\circ}{\stackrel{\infty}{\stackrel{2}{a}}}$ & 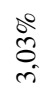 & $\frac{\partial 0}{a}$ & $\begin{array}{l}\text { ڤ̊ } \\
\text { ळे }\end{array}$ & \\
\hline & \multicolumn{2}{|c|}{ 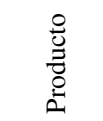 } & $\ll$ & 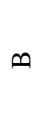 & $u$ & D & II & 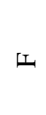 & 0 & $I$ & - & $\sim$ & స్ & $\begin{array}{l}\bar{Q} \\
\overline{\tilde{0}} \\
\tilde{0}\end{array}$ & $\frac{\&}{\tilde{\pi}}$ \\
\hline
\end{tabular}




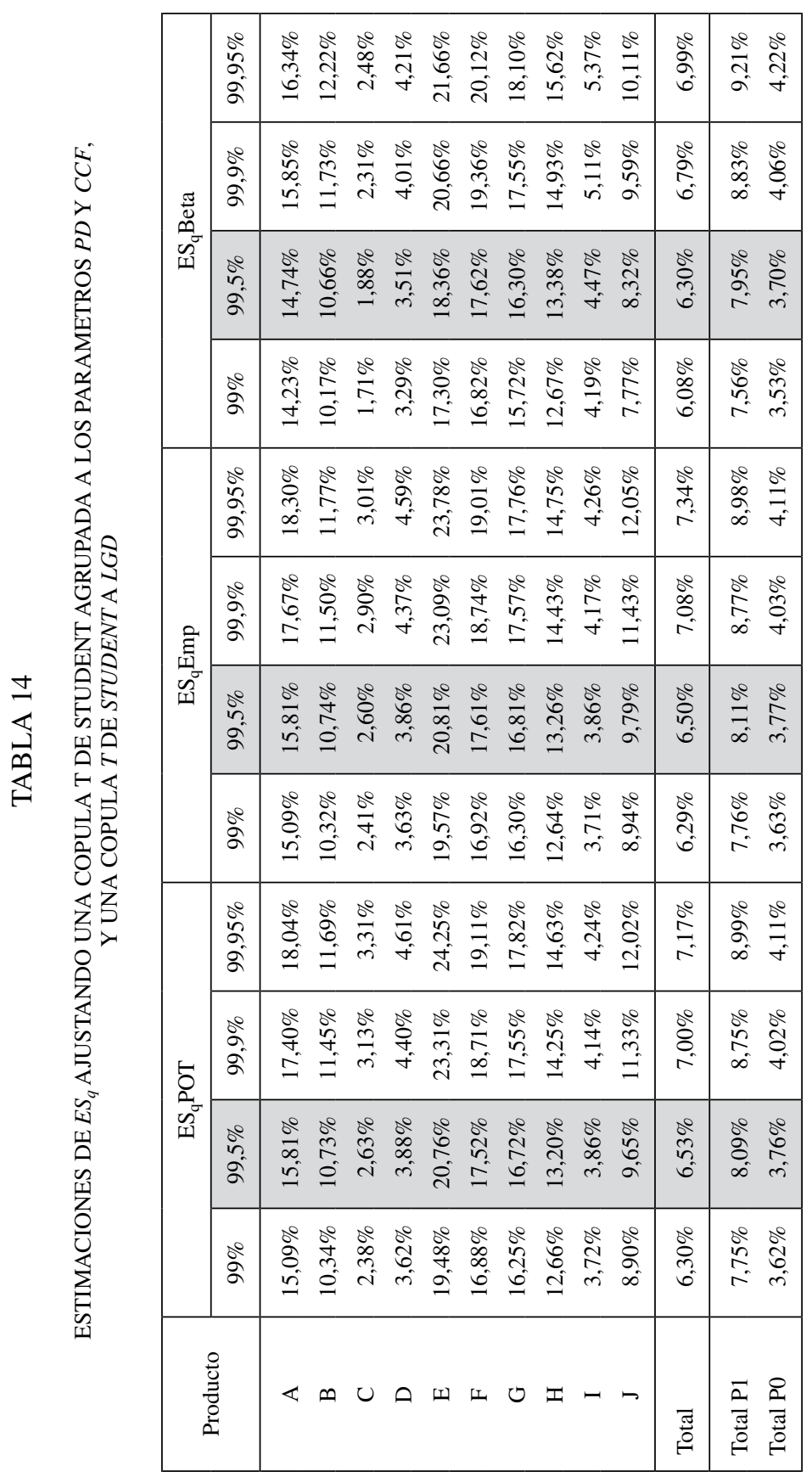


en una muestra finita, lo que produce subestimación del riesgo para valores altos de $q$. La situación es radicalmente distinta cuando se ajusta una distribución Beta (en negro) a las distribuciones de pérdida ya que en ese caso los valores de $\operatorname{VaR}_{q}$ y $E S_{q}$ resultan inferiores incluso a sus correspondientes estimadores empíricos con niveles de confianza superiores al $99 \%$.

\section{FIGURA 4}

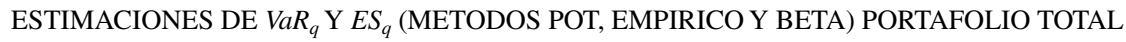

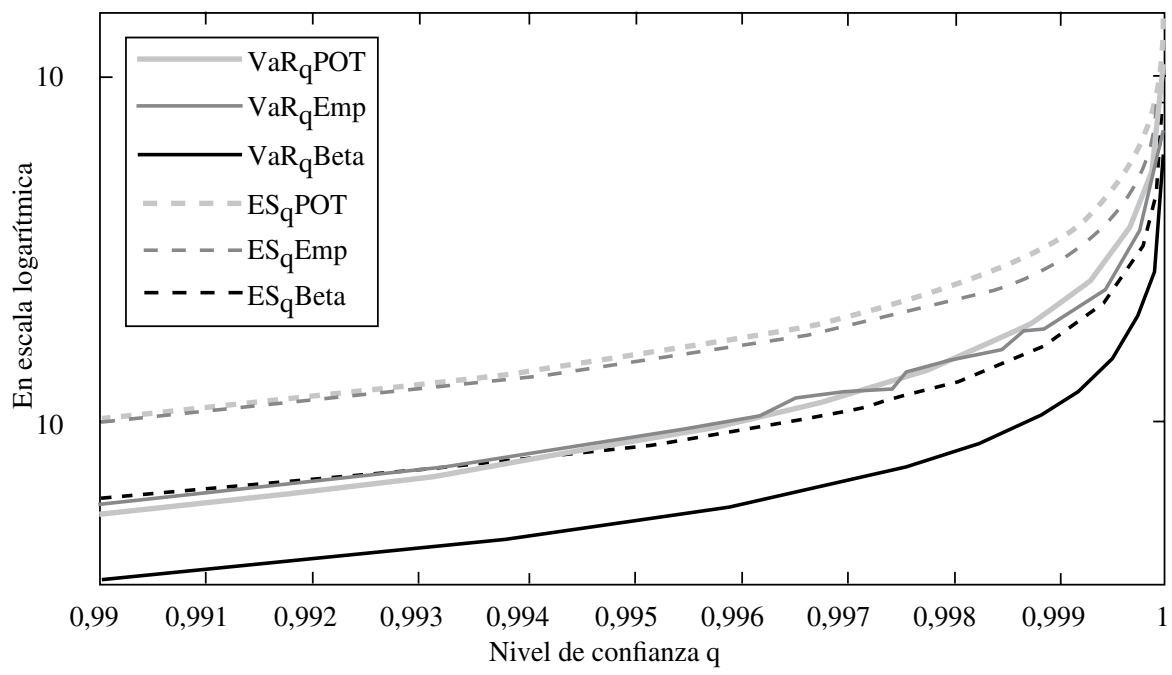

En la Tabla 15 se incluyen los intervalos de confianza del $95 \%$ para los estimadores de las dos medidas de riesgo $V_{a R_{99,5}}$ y $E S_{99,5 \%}$ bajo los métodos POT y Beta. El primer método arroja intervalos de confianza de mayor amplitud que el segundo, y en general los estimadores de $E S_{q}$ presentan mayor variabilidad que el $V_{a} R_{q}$.

\section{ESTIMACIONES DEL CER Y CR BAJO MODELOS TRADICIONALES}

Con el fin de probar la robustez de la metodología propuesta procederemos a comparar sus resultados con los de otros enfoques de medición de riesgo de crédito adoptados por reguladores e instituciones financieras de México en sus análisis de portafolios de consumo. En particular nos concentraremos en el modelo de incumplimiento de un solo factor del tipo KMV/CM y en el modelo de requerimiento de capital regulatorio aceptado por Basilea II (BCBS, 2004) en su enfoque avanzado de calificaciones internas (IRB). 


\section{TABLA 15}

INTERVALOS DE CONFIANZA PARA VAR ${ }_{99,5 \%} \mathrm{Y} \mathrm{ES}_{99,5 \%}$

\begin{tabular}{|c|r|r|r|r|r|r|r|r|}
\hline \multirow{2}{*}{ Producto } & \multicolumn{4}{|c|}{ Método POT } & \multicolumn{3}{c|}{ Ajuste Beta } \\
\cline { 2 - 9 } & \multicolumn{2}{|c|}{$\mathrm{VaR}_{99,5 \%}$} & \multicolumn{2}{|c|}{$\mathrm{ES}_{99,5 \%}$} & \multicolumn{2}{|c|}{$\mathrm{VaR}_{99,5 \%}$} & \multicolumn{2}{|c|}{$\mathrm{ES}_{99,5 \%}$} \\
\hline $\mathrm{A}$ & $14,46 \%$ & $15,34 \%$ & $15,24 \%$ & $16,52 \%$ & $13,76 \%$ & $14,29 \%$ & $14,33 \%$ & $15,36 \%$ \\
$\mathrm{~B}$ & $9,94 \%$ & $10,45 \%$ & $10,37 \%$ & $11,06 \%$ & $9,76 \%$ & $10,21 \%$ & $10,38 \%$ & $10,86 \%$ \\
$\mathrm{C}$ & $2,15 \%$ & $2,50 \%$ & $2,41 \%$ & $2,97 \%$ & $1,56 \%$ & $1,73 \%$ & $1,78 \%$ & $2,09 \%$ \\
$\mathrm{D}$ & $3,48 \%$ & $3,59 \%$ & $3,83 \%$ & $3,98 \%$ & $3,17 \%$ & $3,25 \%$ & $3,48 \%$ & $3,58 \%$ \\
$\mathrm{E}$ & $18,82 \%$ & $19,48 \%$ & $20,46 \%$ & $21,42 \%$ & $16,71 \%$ & $17,28 \%$ & $18,19 \%$ & $18,70 \%$ \\
$\mathrm{~F}$ & $16,45 \%$ & $16,95 \%$ & $17,32 \%$ & $17,92 \%$ & $16,33 \%$ & $16,70 \%$ & $17,50 \%$ & $17,95 \%$ \\
$\mathrm{G}$ & $15,90 \%$ & $16,41 \%$ & $16,45 \%$ & $17,15 \%$ & $15,30 \%$ & $15,67 \%$ & $16,08 \%$ & $16,56 \%$ \\
$\mathrm{H}$ & $11,82 \%$ & $13,77 \%$ & $12,41 \%$ & $14,87 \%$ & $12,01 \%$ & $13,48 \%$ & $12,89 \%$ & $14,88 \%$ \\
$\mathrm{I}$ & $3,61 \%$ & $3,74 \%$ & $3,80 \%$ & $3,97 \%$ & $4,04 \%$ & $4,15 \%$ & $4,40 \%$ & $4,58 \%$ \\
J & $7,95 \%$ & $9,67 \%$ & $8,84 \%$ & $10,95 \%$ & $7,22 \%$ & $8,11 \%$ & $7,81 \%$ & $9,13 \%$ \\
\hline Total & $6,17 \%$ & $6,33 \%$ & $6,46 \%$ & $6,69 \%$ & $5,96 \%$ & $6,06 \%$ & $6,26 \%$ & $6,44 \%$ \\
\hline
\end{tabular}

El modelo del tipo KM/CM es regularmente utilizado para estimar el capital económico de portafolios de créditos revolventes y de consumo (RMA, 2003). Sus parámetros importantes, la correlación de activos (AVC) y de incumplimientos (LC), son usualmente calibrados con información histórica sobre las tasas de incumplimiento. Concretamente, el $C E R_{i}$ (como porcentaje del saldo) de la cubeta $i$ se calcula como

$$
K_{i}=L G D_{i} \cdot C C F_{i} \cdot \Phi\left(\frac{\Phi^{-1}\left(P D_{i}\right)+\sqrt{A V C_{i}} \Phi^{-1}(q)}{\sqrt{1-A V C_{i}}}\right)
$$

donde $q$ es el nivel de confianza y $P D_{i}$ el valor promedio de las correspondientes tasas de incumplimiento observadas.

Los lineamientos actuales en materia de requerimientos mínimos de capital propuestos por el enfoque IRB de Basilea II en riesgo de crédito (ver BCBS, 2005), tienen su base en resultados asintóticos sobre los cuantiles de la función de pérdidas del portafolio bajo el modelo de un solo factor sistémico del tipo KMV/CM (Frey y McNeil, 2003). En particular, el IRB estima el requerimiento de capital regulatorio $C R_{i}$, para cada cubeta $i$ de créditos homogéneos en perfil de riesgo como:

$$
C R_{i}=L G D_{i} \cdot C C F_{i} \cdot \Phi\left(\frac{\Phi^{-1}\left(P D_{i}\right)+\sqrt{\rho} \Phi^{-1}(, 999)}{\sqrt{1-\rho}}\right)
$$

donde el nivel de confianza se fija en $q=0,999$ y la correlación de activos $\rho$ está preestablecida según el tipo de portafolio al que pertenezca la cubeta $(0,04$ para tarjeta de crédito, 0,15 para hipotecario y $0,03\left(\frac{1-e^{-35 P D_{i}}}{1-e^{-35}}\right)+0,16\left(1-\frac{1-e^{-35 P D_{i}}}{1-e^{-35}}\right)$ para el portafolio de 
consumo) independientemente de la estructura específica del portafolio en consideración. Este enfoque regulatorio supone que la correlación de activos está determinada por el parámetro $P D$; sin embargo, el supuesto mismo del modelo de un factor presupone la influencia de la volatilidad de $P D$, especialmente para valores pequeños de ésta. No obstante que la función del parámetro $\rho$ es la de incorporar la independencia entre los activos de los deudores individuales a través de su dependencia individual sobre el estado general de la economía, su determinación mediante reglas fijas puede llevar a mediciones equivocadas del verdadero nivel del riesgo del portafolio.

Al computar las medidas de $C E R$ y $\mathrm{CR}$ con cada uno de los modelos se encuentran los siguientes dos resultados: (1) mientras que el modelo de un factor KMV/CM arroja un $C E R$ menor que el requerimiento de capital regulatorio estipulado por Basilea II, los porcentajes de CR y $C E R$ en ambos modelos son inferiores al $C E R$ registrado por la cópula agrupada. La evidencia es expuesta en la Tabla 16 donde se exhiben los valores del $C E R$ (medido a través de $V a R_{q}$ ) y CR obtenidos con el modelo de un factor y el $\operatorname{VaR}_{q}$ y las reglas de IRB de Basilea II ${ }^{15}$ con el método POT (en donde se consideran densidades kernel con un factor de decaimiento $\lambda=0,97$ ).

TABLA 16

ESTIMACIONES DE CE POR EL MODELO DE UN FACTOR KMV/CM, EL CAPITAL REGULATORIO BAJO EL ENFOQUE IRB DE BASILEA II Y EL CE DEL MODELO COPULA T DE STUDENT AGRUPADA

\begin{tabular}{|c|c|c|c|c|c|c|c|}
\hline \multirow{2}{*}{ Producto } & \multicolumn{2}{|c|}{$\begin{array}{c}\text { Modelo de un factor } \\
\text { KMV/CM }\end{array}$} & \multirow{2}{*}{$\begin{array}{c}\text { Capital } \\
\text { Regulatorio } \\
\text { IRB } \\
\text { avanzado } \\
\text { Basilea II } \\
\end{array}$} & \multicolumn{2}{|c|}{ Cópula t Agrupada } & \multicolumn{2}{|c|}{$\begin{array}{c}\text { Cópula t Agrupada } \\
\lambda=0,97\end{array}$} \\
\hline & $99,5 \%$ & $99,9 \%$ & & $99,5 \%$ & $99,9 \%$ & $99,5 \%$ & $99,9 \%$ \\
\hline A & $12,93 \%$ & $14,07 \%$ & $14,51 \%$ & $14,79 \%$ & $16,45 \%$ & $14,76 \%$ & $16,62 \%$ \\
\hline B & $9,65 \%$ & $10,92 \%$ & $10,64 \%$ & $10,20 \%$ & $11,07 \%$ & $10,48 \%$ & $11,42 \%$ \\
\hline C & $1,00 \%$ & $1,24 \%$ & $0,63 \%$ & $2,29 \%$ & $2,85 \%$ & $2,31 \%$ & $2,71 \%$ \\
\hline $\mathrm{D}$ & $2,16 \%$ & $2,41 \%$ & $2,59 \%$ & $3,52 \%$ & $4,10 \%$ & $3,30 \%$ & $3,85 \%$ \\
\hline $\mathrm{E}$ & $10,43 \%$ & $11,17 \%$ & $13,80 \%$ & $19,00 \%$ & $21,89 \%$ & $17,62 \%$ & $20,80 \%$ \\
\hline $\mathrm{F}$ & $12,71 \%$ & $13,92 \%$ & $14,88 \%$ & $16,66 \%$ & $18,09 \%$ & $16,52 \%$ & $18,22 \%$ \\
\hline $\mathrm{G}$ & $14,92 \%$ & $16,65 \%$ & $15,52 \%$ & $16,09 \%$ & $17,13 \%$ & $16,06 \%$ & $16,20 \%$ \\
\hline $\mathrm{H}$ & $7,17 \%$ & $7,47 \%$ & $9,57 \%$ & $12,46 \%$ & $13,68 \%$ & $12,15 \%$ & $13,17 \%$ \\
\hline I & $2,03 \%$ & $2,11 \%$ & $2,91 \%$ & $3,66 \%$ & $3,99 \%$ & $3,63 \%$ & $4,02 \%$ \\
\hline $\mathrm{J}$ & $4,57 \%$ & $5,09 \%$ & $7,31 \%$ & $8,58 \%$ & $10,32 \%$ & $7,83 \%$ & $9,81 \%$ \\
\hline Total & $5,55 \%$ & $6,08 \%$ & $6,47 \%$ & $6,21 \%$ & $6,74 \%$ & $6,09 \%$ & $6,41 \%$ \\
\hline
\end{tabular}

15 En ambos enfoques los parámetros CCF y LGD se consideran constantes y, en nuestro caso, iguales a sus valores promedios históricos. 
Las diferencias entre los modelos son explicadas por varios hechos. Los valores distintos obedecen a la diversidad de criterios de información y de procedimientos en el tratamiento de las estructuras de dependencia. En efecto aun cuando la construcción de los dos modelos tradicionales es esencialmente la misma, la correlación de activos se calcula sobre bases diferentes; esto es: mientras que el enfoque de un solo factor utiliza información histórica, el enfoque IRB incorpora correlaciones de activos predeterminadas a un nivel de confianza $q$ fijado en 0,999 , como lo muestra la ecuación (11). Y esto produce cifras muy diferentes entre el $C E R$ y $C R$. Pero más importante aún es que ninguno de los dos enfoques modela la estructura de dependencia de los parámetros de riesgo entre cubetas de créditos del mismo producto o entre productos $\mathrm{y}$, en consecuencia, ninguno incorpora los efectos de dependencia conjunta extrema, como en efecto lo hace la cópula t agrupada. Las consecuencias de esas deficiencias redundan en una subestimación de las pérdidas en el área de la cola derecha de las distribuciones subyacentes y en una omisión del efecto de diversificación del portafolio por parte de los dos modelos tradicionales.

\section{CONCLUSIONES: UN BALANCE GENERAL}

El documento presenta un procedimiento de cálculo del CER para un portafolio de créditos al menudeo. El objetivo es desarrollar una metodología en cuatro etapas que facilite el cómputo del $C E R$ en instituciones financieras como las mexicanas. Las etapas para el tratamiento de dicho portafolio son: (1) segmentación en cubetas y productos y estimación de parámetros de riesgo; (2) aplicación de diferentes tipos de estructuras de dependencia multivariadas; (3) pruebas de bondad de ajuste; y (4) comparación con otros métodos de estimación del $C E R$ y $C R$. Con excepción de la primera etapa, las restantes son cruciales para el cálculo adecuado del $C E R$ debido a que su omisión limita los alcances de la metodología.

Las conclusiones principales sostienen que, durante el desarrollo de las etapas (2) y (3), las cópulas elípticas -generalizada y agrupada- de las familias $t$ de Student ofrecen mejores resultados en la modelación del comportamiento de las pérdidas extremas que cualquier otra estructura de dependencia comúnmente utilizada en finanzas, como es el caso de la cópula gaussiana y la de independencia. ${ }^{16}$ En concreto, el ejercicio del punto 3.3 confirma que: a) la cópula $t$ de Student agrupada es la que presenta el mejor ajuste de todas las estructuras de dependencia, pues permite modelar la relación entre productos con diferentes índices de dependencia extrema y, como consecuencia, observar la agregación de las pérdidas del portafolio total y el beneficio del efecto de diversificación; b) las diferencias por producto entre los ajustes de la cópula simétrica y la generalizada no son significativas, aun cuando esta última incorpora parámetros de asimetría en la estructura de dependencia. Finalmente, en la etapa (4) se observa que el modelo de incumplimiento de un solo factor tiende

16 Además de la cópula $t$ agrupada existen otras cópulas relacionadas con la familia $t$ de Student como la t de Valores Extremos y la $t$ de Cola Inferior. Sin embargo, se decidió no incluirlas porque no agregan mayor información al análisis y su manipulación es bastante complicada (Demarta y McNeil, 2004). 
a subestimar el CER. Para la mayoría de los productos, el modelo de un solo factor arroja un $C E R$ menor que el requerimiento de capital regulatorio estipulado por Basilea II, lo cual obedece al hecho de que mientras la correlación de activos utilizada por el primero es calculada con información histórica, en el segundo ésta es fijada con criterios regulatorios y un nivel de confianza predeterminado del 99,9\%. Las estimaciones de CER en el modelo propuesto son, en general, superiores al requerimiento regulatorio que establecen las reglas de capitalización del enfoque más avanzado de IRB. La evidencia de nuestro portafolio sugiere que el capital regulatorio fijado por Basilea II puede resultar insuficiente para cubrir el capital en riesgo del banco bajo estudio al otorgar créditos al menudeo.

Como todo procedimiento estadístico, la metodología aquí propuesta no se encuentra exenta de limitaciones. Para empezar es sabido que las estimaciones de riesgo de crédito en el país están seriamente afectadas por la falta de registros de largo plazo confiables (Márquez, 2006). En nuestro caso, esta carencia afecta seriamente la efectividad de los ajustes de las cópulas o de los métodos de valores extremos debido a las propiedades asintóticas de sus estimadores. No hay duda que con una muestra mayor, la calidad de las medidas de riesgo hubiera mejorado sustancialmente y bajo supuestos más realistas. En particular se hubiera podido utilizar técnicas de series de tiempo para modelar las observaciones históricas de los parámetros de riesgo en lugar de considerarlas variables i.i.d., como se asume en los supuestos (1) y (2) del apartado 3.1. Del mismo modo hubiera sido deseable comparar los resultados de esta metodología con otros modelos propuestos para mejorar la estimación de los parámetros de codependencia (véase Chan-Lau, 2008) o para capturar los eventos extremos sin necesidad de calibrar previamente las codependencias con correlaciones de rango (Segoviano y Goodhart, 2009). En descarga de esta omisión cabe señalar que la implementación de estos modelos en portafolios de créditos al menudeo no es automática ni sencilla, pues hay que tener en mente que considera posiciones crediticias de empresas, no de personas físicas como en nuestro caso, y a un tipo y frecuencia de información que son casi imposibles de obtener para el caso de México. En cualquier caso la comparación queda pendiente como una futura agenda de investigación.

No obstante las deficiencias, el documento constituye un esfuerzo importante en la exploración de métodos que no dependen de modelos de préstamos comerciales para calcular el riesgo de portafolios de créditos de personas físicas. La combinación del uso de cópulas multivariadas con algunos resultados de la TVE es una prueba de que hay manera de tratar portafolios tan diferentes (como son los de préstamos comerciales y al menudeo) con métodos distintos. La mayor virtud de la metodología aquí expuesta es la gran flexibilidad que ofrece al administrador de riesgos para modelar no sólo la dependencia existente entre los factores de riesgo, sino también entre los grupos de créditos y productos del portafolio: un aspecto que no siempre es posible encontrar en los modelos tradicionales ya vistos o en aquellos originalmente diseñados para créditos comerciales. 


\section{REFERENCIAS}

BASEL COMMITTEE ON BANKING SUPERVISION (2004). International Convergence of Capital Measurement and Capital Standards: A Revised Framework, Basel Committee on Banking Supervision. http://www.bis.org/publ/bcbs107.htm

BASEL COMMITTEE ON BANKING SUPERVISION (2005). An explanatory note on the Basel II IRB risk weight functions, Basel Committee on Banking Supervision. http://www.bis.org/bcbs/ irbriskweight.htm

BIGGS, D.; B. DE VILLE y E. SUEN (1991). "A method of choosing multiway partitions for classification and decision trees", Journal of Applied Statistics 18, pp. 49-62.

BOUYE, E.; V. DURRLEMAN; A. NIKEGHBALI; G. RIBOULET y T. RONCALLI (2000). "Copulas for Finance. A Reading Guide and Some Applications”, Financial Econometrics Research Centre, CUBSL, London.

CHAN-LAU, J. (2008). "Default Risk Codependence in the Global Financial System: Was the Bear Stearns Bailout Justified?" http://www.bcentral.cl/conferencias-seminarios/seminarios/index.htm

CROSBIE, P. y J. BOHN (2002). "Modelling Default Risk", KMV working paper.

CROUHY, M.; D. GALAI y R. MARK (2000). "A comparative analysis of current credit risk models", Journal of Banking and Finance 24, pp. 59-117.

DE ANDRADE, F. y L. THOMAS (2004). "Structural Models In Consumer Credit", Risk and Insurance 0407001, EconWPA.

DEMARTA, S. y A. MCNEIL (2004). "The t copula and related copulas”, International Statistical Review 73 (1), pp. 111-129.

DIAZ, A. (2003). "Teoría de Valores Extremos para sucesiones de variables aleatorias dependientes", Tesis de licenciatura, UNAM.

DIKS, C.; W.R. van ZWET; F. TAKENS y J. DEGOEDE (1996). "Detecting differences between delay vector distributions", Physical Review E 53 (3), pp. 2169-2176.

DOWD, K. (2000). "Assessing VaR Accuracy”, Derivatives Quarterly 6 (3), pp. 61-63.

EMBRECHTS, P.; C. KLÜPPELBERG y T. MIKOSH (1997). "Modelling Extremal Events for Insurance and Finance", Springer Verlag, Berlin.

EMBRECHTS, P.; A. MCNEIL y D. STRAUMANN (2002). "Correlation and dependency in risk management: properties and pitfalls", In Risk Management: Value at Risk and Beyond, M. Dempster, Cambridge University Press, pp. 176-223.

FRAHM, G. y M. JUNKER (2003). “Generalized elliptical distributions: models and estimation”, Research Center Caesar Financial Engineering.

FREY, R. y A. MCNEIL (2003). "Dependent defaults in models of portfolio credit risk", Journal of Risk $6(1)$, pp. 59-92.

HULT, H. y F. LINDSKOG (2001). "Multivariate extremes, aggregation and dependence in elliptical distribution", Research paper, RiskLab.

KOLE, E.; K. KOEDIJK y M. VERBEEK (2005). "Testing copulas to model financial dependence", Department of Financial Management, RSM Erasmus University, Rotterdam, The Netherlands.

KOSTADINOV, K. (2005). "Non-parametric estimation of elliptical copulae with application to credit risk", Research paper, Munich University of Technology.

MARQUEZ, J. (2006). Una nueva visión del riesgo de crédito, Limusa, México.

MARQUEZ J. y F. LOPEZ (2006). "Un modelo de riesgo de crédito y su aplicación para realizar una prueba de estrés del sistema financiero mexicano", Estabilidad financiera 10, pp. 25-54.

MATTEN, C. (2000). Managing Bank Capital. Capital Allocation and Performance Measurement, John Wiley \& Sons, New York.

PANCHENKO, V. (2005). "Goodness of fit test for copulas", Physica A 355(1), pp. 176-182.

PERLI, R. y W. NAYDA (2004). "Economic and Regulatory Capital Allocation for Revolving Retail Exposures", Journal of Banking \& Finance 28 (4), pp. 789-809.

RAMIREZ, J. C. (2004). "Usos y limitaciones de los procesos estocásticos en el tratamiento de distribuciones de rendimientos con colas gordas”, Revista de Análisis Económico 19 (1), pp. 51-76.

RMA Capital Working Group (2003). Economic Capital Estimation: Best Practices in Retail Credit, The RMA Journal 85 (7), pp. 68-69.

SAITA, F. (2007). Value at Risk and Bank Capital Management, Academic Press Advanced Finance Series, Elsevier Inc.

SEGOVIANO, M. y C. GOODHART (2009). "Banking Stability Measures", Working Paper 09/4, International Monetary Fund. 


\section{APENDICE}

\section{A1. Estimación de las medidas de riesgo}

Supóngase que $x_{1}, \ldots, x_{n}$ representan las $n$ realizaciones independientes de la variable aleatoria de pérdidas $X$ cuya función de distribución subyacente es $F(x)$. Entonces para un nivel de confianza $0<q<1$ el Valor en Riesgo y el Déficit Esperado de las pérdidas se definen como $\operatorname{VaR}_{q}=\inf \{x \mid F(x) \geq q\}$ y $E S_{q}=\frac{1}{1-q} \int_{q}^{1} \operatorname{VaR} R_{u} d u$, respectivamente.

Los estimadores de $\operatorname{VaR}_{q}$ y $E S_{q}$ bajo el método POT están dados por

$V a R_{q} P O T=u+\frac{\hat{\beta}}{\hat{\xi}}\left(\left((1-q) n / N_{u}\right)^{-\hat{\xi}}-1\right)$ y $E S_{q}=\frac{V a R_{q} P O T+\hat{\beta}-\hat{\xi} u}{1-\hat{\xi}}$

donde $\hat{\beta}$ y $\hat{\xi}$ son los estimadores de máxima verosimilitud (EMV) de la distribución de Pareto Generalizada (DPG) $G_{\xi, \beta}(x)=1-\left(1+\frac{\xi}{\beta} x\right)^{-1 / \xi}$, para $x \geq 0$ si $\xi \geq 0$ o bien para $0 \leq x \leq-\beta / \xi$ si $\xi<0 ;$ y $N_{u}$ denota el número de excesos al umbral $u$ en la muestra. Los estimadores anteriores son consecuencia directa de un resultado central de la TVE que establece que la llamada distribución de excesos $F_{u}(x)=P\{X-u \leq x \mid X>u\}$ se puede aproximar adecuadamente mediante $G_{\xi, \beta(u)}(x)$ para valores grandes de $u$. Para el caso de nuestra aplicación empírica, la selección del umbral u se realiza de tal manera que se minimiza $\frac{1}{N_{u}} \sum_{i=1}^{N_{u}} i^{a} \mid \hat{\xi}_{i}-$ mediana $\left(\hat{\xi}_{1}, \ldots, \hat{\xi}_{N_{u}}\right) \mid$ para valores $0 \leq a<1 / 2$ donde las estimaciones de los paramétros $\xi$ y $\beta$ se estabilizan (ver Embrechts et al., 1997). Los umbrales cercanos al cuantil empírico del $90 \%$ resultan, en general, adecuados para cumplir el criterio anterior.

Si en vez de utilizar la DPG como aproximación a la distribución de los excesos, se ajusta una distribución Beta cuya densidad está dada por $f_{a, b}(x)=x^{a-1}(1-x)^{b-1} / B(a, b), 0<x<1, a, b>0$ donde $B(a, b)=\int_{0}^{1} x^{a-1}(1-x)^{b-1} d x$ es la función beta y los parámetros $a$ y $b$ son estimados por máxima verosimilitud, entonces los estimadores de riesgo deberían ser calculados como:

$$
\operatorname{VaR}_{q} \operatorname{Beta}=F_{\hat{a}, \hat{b}}^{-1}(q) \text { y } \quad \operatorname{ES}_{q} \text { Beta }=\frac{1}{1-q} \int_{V_{\text {VaR }} \text { Beta }}^{1} x f_{\hat{a}, \hat{b}}(x) d x .
$$

Finalmente, si se utiliza la función de distribución empírica $\hat{F}(x)=\frac{1}{n} \sum_{i=1}^{n} 1_{\left\{X_{i} \leq x\right\}}$, para aproximar los estadísticos de orden $X_{(1)}, \ldots, X_{(n)}$ mediante la corrección del tipo 
$\hat{F}^{-1}\left(\frac{j+\delta_{j}}{n+\gamma_{j}}\right)=X_{(j)}$, con $\left(\delta_{j}, \gamma_{j}\right)$ elegidos para tener alguna corrección por continuidad e interpolar para valores $\frac{j-1+\delta_{j}}{n+\gamma_{j}}<q \leq \frac{j+\delta_{j}}{n+\gamma_{j}}$, entonces los correspondientes estimadores empíricos para $\left(\delta_{j}, \gamma_{j}\right)=(-0,5,0)$ son $\operatorname{VaR}_{q} \operatorname{Emp}=x_{(j-1)}+(n q-j+3 / 2)\left(x_{(j)}-x_{(j-1)}\right) \mathrm{y}$ $\left.E S_{q} \operatorname{Emp}=\frac{1}{N_{\operatorname{VaR}_{q} E m p}} \sum_{i=1}^{n} 1_{\left\{x_{i}>\operatorname{VaR} R_{q} E m p\right.}\right\}$

\section{A2. Intervalos de confianza para $\operatorname{VaR}_{q}$ y $E S_{q}$}

El uso de las curvas de log-verosimilitud fiduciales permite determinar intervalos de confianza de $\operatorname{VaR}_{q}$ y $E S_{q}$. para los estimadores obtenidos por los métodos POT y Beta. Si cualquiera de los estimadores dados por las ecuaciones (13) y (14) se denota como $\phi=g\left(\theta_{1}, \theta_{2}\right)$ donde $\theta_{1}$ y $\theta_{2}$ son los parámetros del modelo, entonces la función de verosimilitud reparametrizada $L\left(\phi, \theta_{1}\right)$ permite contrastar la hipótesis nula

$H_{0}: \phi=\phi_{0}$ contra la alternativa $H_{1}: \phi \neq \phi_{0}$ mediante la estadística del cociente de verosimilitudes $\lambda=\frac{\sup _{\theta_{1}} L\left(\phi_{0}, \theta_{1}\right)}{\sup _{\phi, \theta_{1}} L\left(\phi, \theta_{1}\right)}$. Bajo $H_{0}$ se puede demostrar que $-2 \log (\lambda) \sim \chi_{(1)}^{2}$ y que dado el EMV del modelo restringido bajo la hipótesis nula $\left(\hat{\theta}_{10}\right)$ y los correspondientes EMV del modelo irrestricto $\left(\hat{\theta}_{1}, \hat{\theta}_{2}\right)$, se rechaza $H_{0}$ al nivel de confianza $(1-\alpha)$ si $-2 \cdots \log L\left(\phi_{0}, \hat{\theta}_{10}\right)-2 \log L\left(g\left(\hat{\theta}_{1}, \hat{\theta}_{2}\right), \hat{\theta}_{1}\right)>\chi_{(1,1-\alpha)}^{2}$ donde $\chi_{(1,1-\alpha)}^{2}$ es el cuantil de nivel $1-\alpha$ de una distribución $\chi_{(11)}^{2}$. Entonces el intervalo de confianza fiducial para $\phi$ está dado por

$$
\left\{\phi_{0} \mid \log L\left(\phi_{0}, \hat{\theta}_{10}\right) \geq \log L\left(g\left(\hat{\theta}_{1}, \hat{\theta}_{2}\right), \hat{\theta}_{1}\right)-0,5 \chi_{(1,1-\alpha)}^{2}\right\} .
$$

\section{A3. Algoritmos de estimación para Meta-distribuciones Elípticas}

Entre los numerosos procedimientos estadísticos diseñados para tratar con distribuciones elípticas, son pocos los que ajustan cópulas elípticas independientemente de sus marginales, como el desarrollado por Kostadinov (2005) y que aparece resumido en el algoritmo 1. El método permite estimar robustamente las medidas de dependencia $\rho_{\tau}$ y $\lambda$, así como el índice de la cola $\alpha$ y los parámetros de la cópula $t$ de Student agrupada. Para el caso de las cópulas elípticas generalizadas se propone el algoritmo 2 para estimar $\mu$ y $\Sigma$ de acuerdo con el procedimiento sugerido por Frahm y Junker (2003). 
En ambos algoritmos se considera que las realizaciones de $n$ copias independientes del vector aleatorio $\boldsymbol{X}$ (el conjunto de los datos) están denotadas por

$$
\left\{\left(x_{1 t}, \ldots, x_{d t}\right)^{\prime}\right\}_{t=1}^{n} \equiv\left[\begin{array}{cccc}
x_{11} & x_{12} & \cdots & x_{1 n} \\
x_{21} & \ddots & & \vdots \\
\vdots & & \ddots & \vdots \\
x_{d 1} & \cdots & \cdots & x_{d n}
\end{array}\right]=\left[\begin{array}{llll}
x_{\cdot l} & x_{\cdot 2} & \cdots & x_{\cdot n}
\end{array}\right]
$$

Algoritmo 1

(i) Determinar las correlaciones tau de Kendall de cada pareja $\left(X_{i}, X_{j}\right)$ mediante el estimador $\hat{\rho}_{\tau}^{(n)}\left(X_{i}, X_{j}\right)=\left(\begin{array}{l}n \\ 2\end{array}\right)^{-1} \sum_{k>l} \operatorname{sign}\left[\left(x_{i k}-x_{i l}\right)\left(x_{j k}-x_{j l}\right)\right]$;

(ii) Calcular $\hat{\rho}_{i j}=\operatorname{sen}\left(\frac{\pi}{2} \hat{\rho}_{\tau}^{(n)}\left(X_{i}, X_{j}\right)\right)$ para estimar así la matriz de correlación $\left(\rho_{i j}\right)$;

(iii) Estimar los coeficientes de dependencia de la cola inferior $\hat{\Lambda}^{(n)}=\left(\lambda_{i j}\right)_{i, j=1}^{d}$ como

$$
\lambda_{i j}^{n} \equiv \hat{\lambda}_{l}^{(n, r)}\left(X_{i}, X_{j}\right)=\frac{1}{n} \sum_{k=1}^{n} \frac{\sqrt{2}}{r} 1_{\left\{Q_{k}<r\right\}} \operatorname{sen}\left(2 \phi_{k}\right),
$$

donde para $k=1, \ldots, n$ las cantidades $\left(Q_{k}, \phi_{k}\right)$ satisfacen las ecuaciones $\hat{F}_{i}\left(x_{i k}\right)=Q_{k} \operatorname{sen} \phi_{k}, \hat{F}_{j}\left(x_{j k}\right)=Q_{k} \cos \phi_{k}$, donde cada $\hat{F}_{i}$ es la f.d. empírica de la componente $X_{i}$;

(iv) Estimar el índice de la cola $\alpha$ de la v.a. espectral $R$ como

$$
\hat{\alpha}^{(n)}=\underset{\alpha>0}{\arg \min }\left\|\lambda\left(\alpha, \frac{\pi}{4}\left(1-\hat{\rho}_{i j}\right)\right)_{i j}-\hat{\Lambda}^{(n)}\right\|_{\times},
$$

donde $L\left(\alpha, \hat{\rho}_{\tau}^{(n)}\right)$ es la matriz $\lambda\left(\alpha, \frac{\pi}{4}\left(1-\hat{\rho}_{i j}\right)\right)_{i j}$, la función $\lambda(\alpha, x)$ está definida como $\lambda(\alpha, x)=\frac{\int_{x}^{\frac{\pi}{2}} \cos ^{\alpha} t d t}{\int_{0}^{\frac{\pi}{2}} \cos ^{\alpha} t d t} \mathrm{y}\|A\|_{\times}=\sum_{i, j=1}^{d} A_{i j}^{2}$ define la norma en $L^{2}$ definida sobre $M_{d \times d}(\Re)$. 


\section{Algoritmo 2}

(i) Estimar los parámetros $\left(\rho_{i j}\right)$ y $\alpha$ de una distribución Elíptica Generalizada sobre el vector $\boldsymbol{X}$ usando el Algoritmo 1;

(ii) Ajustar a cada componente de $\boldsymbol{X}$ alguna f.d. marginal $F_{j}{ }^{*}$ continua. Si por ejemplo, el número de observaciones n no es muy grande, se puede utilizar un kernel de suavización;

(iii) Simular observaciones $y_{1}, \ldots, y_{N}$ de un vector $\boldsymbol{Y}$ cuya representación está dada por $Y=\mu+R A U^{(k)}$, utilizando las estimaciones de los parámetros anteriores y estructura de dependencia de dicha representación (bajo la especificación de la f.d. de la v.a. espectral $R$ );

(iv) Construir observaciones $\hat{u}_{1}, \ldots, \hat{u}_{N}$ de la cópula empírica con las observaciones simuladas $y_{1}, \ldots, y_{N}$, a saber, $\hat{u}_{i}=\left(U_{i 1}, \ldots, U_{i d}\right)^{\prime}=\left(\hat{F}_{1}\left(y_{i 1}\right), \ldots, \hat{F}_{d}\left(y_{i d}\right)\right)^{\prime}$ donde $\hat{F}_{j}$ es un estimador de la f.d. de $Y_{j}$, por ejemplo, se puede usar una variante de la f.d. empírica $\hat{F}_{j}(x)=\frac{1}{n+1} \sum_{i=1}^{n} 1_{\left\{y_{i j} \leq x\right\}}$.

(v) Con las observaciones simuladas $\hat{u}_{1}, \ldots, \hat{u}_{N}$, las cuales incorporan la estructura de dependencia de la cópula $C$, construir $\hat{x}_{j}=F_{j}^{*-1}\left(\hat{u}_{j}\right), j=1, \ldots, N$. Los vectores $\hat{x}_{1}, \ldots, \hat{x}_{N}$ son observaciones simuladas del vector original $\boldsymbol{X}$.

\section{A4. Prueba de bondad de ajuste para cópulas}

En este apartado se presenta una prueba de bondad de ajuste no paramétrica desarrollada por Panchenko (2005) que está diseñada tanto para el caso de cópulas bivariadas como multivariadas y, además, está basada en una medida de divergencia que fue introducida por Diks et al. (1996). Dicha prueba puede ser aplicada a cualquier forma funcional de la cópula pues no requiere el uso de los estimadores de los parámetros.

Si se define el producto interno entre dos funciones integrables $f_{1}$ y $f_{2}$ mediante la forma bilineal $\left\langle f_{1}\left|k_{d}\right| f_{2}\right\rangle=\iint k_{d}\left(s_{1}, s_{2}\right) f_{1}\left(s_{1}\right) f_{2}\left(s_{2}\right) d s_{1} d s_{2}$, donde $s_{1}, s_{2} \in \Re^{d}$ y $k_{d}\left(s_{1}, s_{2}\right)=e^{-\frac{\left\|s_{1}-s_{2}\right\|^{2}}{2 d h}}$ es el kernel gaussiano, $\|\cdot\|$ denota la norma euclidiana en $\mathfrak{R}^{d}$ y $h>0$ es un parámetro de suavizamiento.

De acuerdo con esta prueba, la distancia al cuadrado $Q$ entre $f_{1}$ y $f_{2}$ se define mediante $Q=\left\langle f_{1}-f_{2}\left|k_{d}\right| f_{1}-f_{2}\right\rangle$, que por propiedades básicas de producto interno se puede reescribir como $Q=Q_{11}-2 Q_{12}+Q_{22}$, donde $Q_{i j}=\left\langle f_{i}\left|k_{d}\right| f_{j}\right\rangle$. Cada término se puede estimar consistentemente usando las llamadas estadísticas $\mathrm{V}$ dadas por 
$Q_{i j}=\frac{1}{n^{2}} \sum_{t_{1}=1}^{n} \sum_{t_{2}=1}^{n} k_{d}\left(s_{1}^{t_{1}}, s_{2}^{t_{2}}\right)$, donde $\boldsymbol{s}^{t}$ denota una realización del vector aleatorio $s$ al tiempo $t$.

Siguiendo la notación dada en (16), dadas $\boldsymbol{x}_{._{1}}, \boldsymbol{x}_{\cdot 2}, \cdots, \boldsymbol{x}_{{ }_{n}}$ realizaciones de un vector aleatorio $\boldsymbol{X}$, se construyen las correspondientes observaciones $S_{1}$ de la cópula empírica de acuerdo con el paso (iv) del Algoritmo 2.

Sea una cierta cópula $C_{\theta}$ cuyos parámetros han sido estimados por $\hat{\theta}$ mediante algún método estadístico, y sea $S_{2} \equiv\left(S_{2}^{t}\right)_{t=1}^{n}$. Dado que la teoría asintótica para la estadística $Q$ está aún en desarrollo, para contrastar $H_{0}: S_{1}=S_{2}$ vs $H_{1}: S_{1} \neq S_{2}$, se puede utilizar la técnica de bootstrap paramétrico. Para ello, se simulan $m$ muestras $S_{1}^{*}, \ldots, S_{m}^{*}$ de la cópula $C_{\hat{\theta}}$ y se calculan los estimadores $\hat{Q}=\hat{Q}\left(S_{1}, S_{2}\right)$ y $\hat{Q}_{j}^{*}=\hat{Q}\left(S_{2}, S_{j}^{*}\right) j=1, \ldots, m$. Entonces un estimador del $p$-value de la prueba está dado por $\hat{p}=\frac{1}{1+m}\left(1+\sum_{j=1}^{m} 1_{\left\{\hat{Q} \geq \hat{Q}_{j}^{*}\right\}}\right)$, y se rechaza $H_{0}$ si $\hat{p} \leq \alpha$, donde $\alpha$ es el nivel de significancia de la prueba. 